\title{
4
}

\section{Integral Sliding Modes with Block Control of Multimachine Electric Power Systems}

\author{
Héctor Huerta, Alexander Loukianov and José M. Cañedo \\ Centro de Investigación y de Estudios Avanzados del Instituto Politécnico Nacional, \\ Unidad Guadalajara \\ Jalisco, México
}

\section{Introduction}

Over last 15 years the problem of rotor angle stability of electric power systems (EPS) has received a great attention. A fundamental problem in the design of feedback controllers for EPS is that of robust stabilizing both rotor angle and voltage magnitude, and achieving a specified transient behavior. Robustness implies operation with adequate stability margins and admissible performance level in spite of plant parameters variations and in the presence of external disturbances.

The EPS have nonlinearities and are subject to variations as a result of a change in the systems loading and/or configuration. Then, the EPS are modeled as complex large-scale nonlinear systems and the generators may be interconnected over several kilometers in very large power systems. Thus, the controller design is a challenging problem. A complete centralized control scheme could be difficult to implement in EPS, due to the reliability and distortion in information transfer. On the other hand, accurate prediction of system responses and system robustness to disturbances under different operation conditions are guarantee by robust decentralized control schemes. The decentralized controllers are locally implemented, so do not need system information communication among subsystems. In each subsystem, the effects of the other subsystems are considered as a disturbance. To design decentralized control schemes for EPS, a controller is designed for each generator connected to the system.

The control schemes of power systems are commonly based on reduced order linearized model and classical control algorithms that ensure asymptotic stability of the equilibrium point under small perturbations (Anderson \& Fouad, 1994, DeMello \& Concordia, 1969). Improvements on linear techniques have been analyzed in (Wang et al., 1998, Djukanovic et at., 1998a, Djukanovic et al., 1998b). Nevertheless, these controllers have been designed by using linear models. To analyze the EPS entire operation region, nonlinear control design techniques are more appropriate. Various nonlinear techniques have been implemented, e.g., control based on direct Lyapunov method (Machowsky et al., 1999), feedback linearization (FL) technique (Akhkrif, et al, 1999, Wu \& Malik, 2006, ) including backstepping (Jung et al., 2005 King et al., 1994), intelligent neural networks (Venayagamoorthy et al., 2003, Mohagheghi et al., 2007), fuzzy logic (Yousef \& Mohamed, 2004) and normal form analysis (Kshatriya, et al., 2005, Liu et al., 2006). 
All of the mentioned controllers provide larger stability margins with respect to traditional ones. But these control schemes were designed for reduced order plant. The unmodelled electrical dynamics can affect the electromechanical dynamics in case of large perturbations. The detailed 7-th order model of synchronous machine (five equations for electrical dynamics and two for mechanical dynamics) has been considered and a nonlinear controller using this model and FL technique has been designed to enhance transient stability (Akhkrif, et al., 1999). The proposed nonlinear control law is a function of all plant parameters and disturbances. In practice some of these parameters are subjected to variations as a result of a change in the system loading and/or configuration. Since the detailed model is so involved, a direct use of the FL technique results in a computationally expensive control algorithm. Moreover, this control scheme does not take into account practical limitation on the magnitude of the excitation voltage, and an observer design problem was not solved.

On the other hand, sliding mode control (SMC), (Utkin, et al., 1999) is one of the most effective strategies to deal with robust nonlinear controllers. SMC enables high accuracy and robustness to disturbances and plant parameter variations. Moreover, the control variables of the basic sliding mode control law rapidly switch between extreme limits, which are ideal for the direct operation of the switched mode power converters of synchronous generators. Sliding mode controllers for power systems have been designed in (Dash et al., 1996, Bandal et al., 2005), however for reduced order plants only, the best of our knowledge. Application of these controllers to full order plant would cause undesirable chattering, since unmodelled dynamics can be excited.

In (Loukianov et al., 2004) it was designed a sliding mode controller to regulate the terminal voltage and power angle for a single machine infinite-bus system, based on the eighth order generator model (two equations for mechanical dynamics and six equations for electrical ones for thermo electrical power system). In this case, an information about the power angle reference, $\delta_{r e f}$, is required. To overcome this restriction, in (Loukianov et al., 2006) a decentralized robust sliding mode control scheme was proposed to regulate the voltages and stabilize the speed in a multi machine power system.

In this paper an eighth order model for each generator of the multimachine power systems is considered. Sliding mode controller is designed by using the combination of three techniques: block control (Loukianov, 1998), integral sliding mode control (Utkin et al., 1999), and nested sliding mode control (Adhami-Mirhosseini and Yazdanpanah, 2005). The block control technique is used to design a nonlinear sliding surface in such a way that the sliding mode dynamics are represented by a linear system with desired eigenvalues. The integral sliding mode control combined with nested control technique are applied to reject perturbations. The controller designed in this way is computationally low demanding and takes into account structural constraints of the control input. The main feature of the proposed control scheme is robustness with respect to the both matched and unmatched perturbations and only local information is required. Moreover, a nonlinear observer for the unmeasureable estates of the systems such as the rotor fluxes of the generators is presented. This chapter is organized as follows. Section 2 presents a general mathematical description of the EPS (nonlinear eight order electrical generator, electrical network and loads models). Section 3 deals with the problem of nonlinear robust controller for the class of the nonlinear systems represented in the nonlinear block controllable form, the Integral Sliding Modes with Block Control technique is analyzed. Section 4 shows the design of a nonlinear robust 
control scheme for EPS, as well as a generator rotor fluxes observer. The results of the simulations in an equivalent of the WSCC, that illustrates the properties of the controller designed, can be found in section 5 , followed by conclusions in section 6 .

\section{EPS Model}

This section copes with the mathematical description of the EPS. The multimachine EPS model considers the generators model, the electrical network model and loads.

\subsection{Generator model}

The electrical dynamics comprised the field winding, rotor and stator windings, after the Park's transformation, can be expressed as follows (Anderson \& Fouad, 1994):

$$
\left[\begin{array}{l}
d \boldsymbol{\lambda}_{1} / d t \\
d \mathbf{i} / d t
\end{array}\right]=\mathbf{A}(\omega) \cdot\left[\begin{array}{l}
\lambda_{1} \\
\mathbf{i}
\end{array}\right]+\mathbf{T}\left[\begin{array}{l}
\mathbf{v}_{1} \\
\mathbf{v}
\end{array}\right]
$$

where $\lambda_{1}=\left(\lambda_{f}, \lambda_{g}, \lambda_{k d}, \lambda_{k q}\right)^{T}, \mathbf{i}=\left(i_{d}, i_{q}\right)^{T}, \mathbf{v}=\left(v_{d}, v_{q}\right)^{T}, \mathbf{v}_{1}=\left(v_{f}, 0,0,0\right)^{T}, \lambda_{f}$ is the field flux, $\lambda_{k d}, \lambda_{k q}$ and $\lambda_{g}$ are the direct-axis and quadrature-axis damper windings fluxes respectively, $i_{d}$ and $i_{q}$ are the stator currents, $\omega$ is the angular speed, $v_{f}$ is the excitation control input, $v_{d}$ and $v_{q}$ are the direct-axis and quadrature-axis terminal voltages, respectively. The matrices $\mathbf{A}(\omega)=-\mathbf{T}\left[\mathbf{R} \cdot \mathbf{L}^{-1}+\mathbf{W}(\omega)\right] \mathbf{T}^{-1}, \mathbf{T}, \mathbf{R}, \mathbf{L}$ and $\mathbf{W}(\omega)$ are defined in Appendix.

The complete mathematical description includes also the swing equation given by

$$
\begin{aligned}
& d \delta / d t=\omega-\omega_{b} \\
& d \omega / d t=\left(\omega_{b} / 2 H\right)\left(T_{m}-T_{e}\right)
\end{aligned}
$$

where $\delta$ is the power angle, $\omega_{b}$ is the rated synchronous speed, $H$ is the inertia constant, $T_{m}$ is the mechanical torque applied to the shaft, and $T_{e}$ is the electromagnetic torque, expressed in terms of the linked fluxes and currents as follows:

$$
T_{e}=a_{1} \lambda_{f} i_{q}-a_{2} \lambda_{g} i_{d}+a_{3} \lambda_{k d} i_{q}-a_{4} \lambda_{k q} i_{d}-a_{5} i_{d} i_{q}
$$

where $a_{1}, \ldots, a_{5}$ are constants defined in Appendix. The mechanical torque $T_{m}$ it is assumed to be a slowly varying and bounded function of time. Thus:

$$
\dot{T}_{m}=0 .
$$

Since the multimachine EPS has at least one more differential equation than is needed to solve the system, then, it is possible to define the angle relative to the generator 1 of the form:

$$
\hat{\delta}_{i}=\delta_{i}-\delta_{1}, \quad i=1,2, \ldots, n
$$


where $n$ is the number of generators in the system. Thus

$$
\frac{d \hat{\delta}_{1}}{d t}=0, \quad \frac{d \hat{\delta}_{i}}{d t}=\omega_{i}-\omega_{1}, \quad i=2,3, \ldots, n
$$

From (1)-(5), the nonlinear state-space presentation of the $i^{\text {th }}$ generator in the multimachine power system is derived of the form

$$
\begin{gathered}
{\left[\begin{array}{c}
\dot{\mathbf{x}}_{1 i} \\
\dot{\mathbf{x}}_{2 i}
\end{array}\right]=\left[\begin{array}{c}
\mathbf{f}_{1 i}\left(\mathbf{x}_{i}, \mathbf{i}_{i}\right) \\
\mathbf{f}_{2 i}\left(\mathbf{x}_{i}, \mathbf{i}_{i}\right)
\end{array}\right]+\left[\begin{array}{c}
\mathbf{b}_{1 i} \\
0
\end{array}\right] v_{f i}+\left[\begin{array}{c}
\mathbf{g}_{i}\left(\mathbf{x}_{i}, \mathbf{i}_{i}, T_{m i}\right) \\
0
\end{array}\right]} \\
\mu \frac{d}{d t} \mathbf{i}_{i}=\mathbf{A}_{z i}\left(x_{2 i}\right) \mathbf{i}_{i}+\mathbf{f}_{z i}\left(\mathbf{x}_{i}\right)+\mathbf{b}_{z i} v_{f i}+\mathbf{H}_{i} \mathbf{v}_{i}
\end{gathered}
$$

where $\mu=1 / \omega_{b}$ is a small parameter, $\mathbf{x}_{i}=\left(\mathbf{x}_{1 i} \mathbf{x}_{2 i}\right)^{T}, \mathbf{x}_{1 i}=\left(x_{1 i}, x_{2 i}, x_{3 i}\right)^{T}=\left(\hat{\delta}_{i}, \omega_{i}, \lambda_{f i}\right)^{T}$,

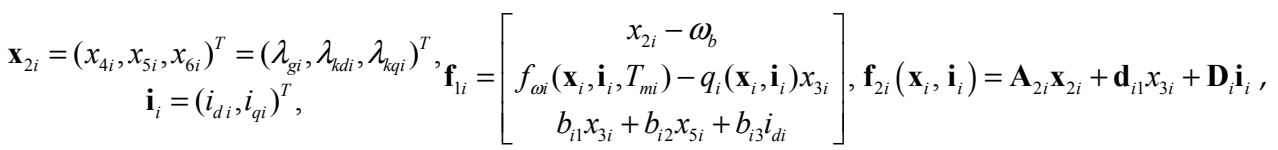
$f_{\omega i}(\cdot)=-d_{m i}\left(-a_{i 2} x_{4 i} i_{d i}+a_{i 3} x_{5 i} i_{q i}-a_{i 4} x_{6 i} i_{d i}+a_{i 5} i_{d i} i_{q i}\right), q_{i}(\cdot)=a_{i 1} d_{m_{i}} i_{q i}, d_{m}=\frac{\omega_{b}}{2 H}$, $\mathbf{d}_{i 1}=\left[\begin{array}{c}0 \\ 0 \\ d_{i 1}\end{array}\right], \mathbf{D}_{i}=\left[\begin{array}{cc}0 & c_{i 3} \\ d_{i 3} & 0 \\ 0 & e_{i 3}\end{array}\right], \mathbf{A}_{2 i}=\left[\begin{array}{ccc}c_{i 1} & 0 & c_{i 2} \\ 0 & d_{i 2} & 0 \\ r_{i 1} & 0 & r_{i 2}\end{array}\right] \mathbf{b}_{1 i}=\left[\begin{array}{l}0 \\ 0 \\ 1\end{array}\right], \mathbf{A}_{z i}=\left[\begin{array}{cc}h_{i 7} & k_{i 6} x_{2 i} \\ h_{i 6} x_{2 i} & k_{i 7}\end{array}\right], \mathbf{H}_{i}=\left[\begin{array}{cc}h_{i 1} & 0 \\ 0 & k_{i 1}\end{array}\right]$, $\mathbf{b}_{z i}=\left[\begin{array}{c}0 \\ h_{i 8}\end{array}\right], \mathbf{f}_{z i}\left(\mathbf{x}_{i}\right)=\left[\begin{array}{l}h_{i 2} x_{3 i}+h_{i 3} x_{5 i}+h_{i 4} x_{2 i} x_{4 i}+h_{i 5} x_{2 i} x_{6 i} \\ k_{i 2} x_{4 i}+k_{i 3} x_{6 i}+k_{i 4} x_{2 i} x_{3 i}+k_{i 5} x_{2 i} x_{5 i}\end{array}\right], \mathbf{v}_{i}=\left[v_{d i}, v_{q i}\right]^{T}$,

$\mathbf{g}_{i}\left(\mathbf{x}_{i}, \mathbf{i}_{i}, T_{m i}\right)=\left[0, g_{2 i}\left(\mathbf{x}_{i}, \mathbf{i}_{i}, T_{m i}\right), 0\right]^{T}$. The perturbation term $g_{2 i}(\cdot)$ includes variations of the generator parameters in the function $f_{\omega i}(\cdot)$ and the mechanical torque $T_{m i}$ (external disturbance), i. e.

$$
g_{2 i}(\cdot)=d_{m} T_{m i}-\left[\Delta a_{i 2} x_{4 i} i_{d i}+\Delta a_{i 3} x_{5 i} i_{q i}+\Delta a_{i 4} x_{6 i} i_{d i}+\Delta a_{i 5} i_{d i} i_{q i}\right], a_{i j}=a_{i j, n}+\Delta a_{i j}, j=2, \ldots, 5,
$$

where $a_{i j, n}$ and $\Delta a_{i j}$ are the nominal value and variation, respectively, of the parameter $a_{i j}$. Moreover $\operatorname{rank}\left\{\mathbf{A}_{z i}\right\}=2$ for all admissible values of $x_{2 i}$.

To neglect the fast dynamics in the electric networks that in turn permits to simplify and simulate the complete power system by a differential algebraic equation (DAE) (Anderson \& Fouad, 1994) we use the singular perturbation technique (Khalil, 1996). Thus, setting $\mu=0$ in (7) results in

$$
0=\mathbf{A}_{z i}\left(x_{2 i}\right) \mathbf{i}_{i}+\mathbf{f}_{z i}\left(\mathbf{x}_{i}\right)+\mathbf{H}_{i} \mathbf{v}_{i}
$$

The solution of (8) for $\mathbf{i}_{i}$ is calculated as 


$$
\mathbf{i}_{i}=\mathbf{g}_{z i}\left(\mathbf{x}_{i}, \mathbf{v}_{i}\right)
$$

where $\mathbf{g}_{z i}=-\mathbf{A}_{z i}^{-1}\left(x_{2 i}\right)\left(\mathbf{f}_{z i}\left(\mathbf{x}_{i}\right)+\mathbf{H}_{i} \mathbf{v}_{i}\right)$. Finally, equations (6) and (9) give the following DAE system for the $i^{\text {th }}$ generator:

$$
\begin{gathered}
\dot{\mathbf{x}}_{1 i}=\mathbf{f}_{1 i}\left(\mathbf{x}_{i}, \mathbf{i}_{i}, T_{m i}\right)+\mathbf{b}_{1 i} v_{f i}+\mathbf{g}_{i}\left(\mathbf{x}_{i}, \mathbf{i}_{i}, T_{m i}\right) \\
\dot{\mathbf{x}}_{2 i}=\mathbf{f}_{2 i}\left(\mathbf{x}_{i}, \mathbf{i}_{i}\right) \\
\mathbf{i}_{i}=\mathbf{g}_{z i}\left(\mathbf{x}_{i}, \mathbf{v}_{i}\right) .
\end{gathered}
$$

\subsection{Electrical network model}

Since the fast dynamics reduction for the generator was achieved in the last subsection, it is possible to neglect the dynamics of the loads and transmission lines. Then, considering the loads as constant impedances, the electrical network can be modeled using the phasorial nodal method. Moreover, all the nodes, except for the generator ones, can be reduced (Kron's reduction). Therefore the network algebraic equation can be expressed as (Anderson \& Fouad, 1994)

$$
\overline{\mathbf{I}}=\overline{\mathbf{Y}}\left(\delta_{1}, \ldots, \delta_{n}\right) \overline{\mathbf{V}}
$$

where $\quad \overline{\mathbf{V}}=\left[v_{d 1}+j v_{q 1}, \cdots, v_{d n}+j v_{q n}\right]^{T}$ and $\quad \overline{\mathbf{I}}=\left[i_{d 1}+j i_{q 1}, \cdots, i_{d n}+j i_{q n}\right]^{T}$ are the complex terminal generators voltages and currents, respectively, $\overline{\mathbf{Y}}(\cdot)$ is the reduced transformed admittance matrix and its entry $j k$ is given by:

$$
\overline{\mathbf{Y}}_{j k}=\mathbf{Y}_{j k} e^{\left(\delta_{j}-\delta_{k}\right)}
$$

with the elements $\mathbf{Y}_{j k}$ calculated by using the nodal method. It is more convenient to express the equation (13) of the form

$$
\mathbf{I}=\tilde{\mathbf{Y}}\left(\delta_{1}, \ldots, \delta_{n}\right) \mathbf{V}, \tilde{\mathbf{Y}}(\cdot) \in R^{2 n \times 2 n}
$$

where $\quad \mathbf{V}=\left[v_{d 1}, v_{q 1}, \ldots, v_{d n}, v_{q n}\right]^{T}$ and $\quad \mathbf{I}=\left[\mathbf{i}_{1}^{T} \ldots \mathbf{i}_{n}^{T}\right]^{T}=\left[i_{d 1}, i_{q 1}, \ldots, i_{d n}, i_{q n}\right]^{T}$ are the phasors components of the voltages and currents, respectively. Thus, the multimachine EPS model is given by (10)-(12) and (14). It is important to note that the vector I coincides with the generator currents $i_{d i}$ and $i_{q i}$.

\section{Integral Sliding Modes with Block Control}

The Integral Sliding Modes with Block Control (ISM) technique (Huerta-Avila et al., 2007a, Huerta-Avila et al., 2007b) is shown in this section. The description of the ISM is presented in generic terms to show the generality of the approach. In the next section a robust controller for the electrical power system will be designed by using this methodology. 


\subsection{Problem statement}

In this work, the class of nonlinear systems presented in the NBC (nonlinear block controllable) form is studied. The NBC form consist of $r$ blocks (Loukianov, 1998):

$$
\begin{aligned}
\dot{\mathbf{x}}_{i} & =\mathbf{f}_{i}\left(\overline{\mathbf{x}}_{i}\right)+\mathbf{B}_{i}\left(\overline{\mathbf{x}}_{i}\right) \mathbf{x}_{i+1}+\mathbf{g}_{i}(t, \mathbf{x}) \\
\dot{\mathbf{x}}_{r} & =\mathbf{f}_{r}(\mathbf{x})+\mathbf{B}_{r}(\mathbf{x}) \mathbf{u}+\mathbf{g}_{r}(t, \mathbf{x}), \quad i=1, \ldots, r-1, \\
\mathbf{y} & =\mathbf{x}_{1}
\end{aligned}
$$

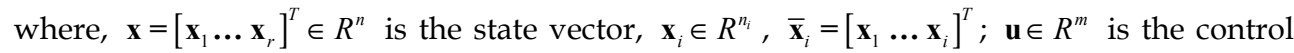
vector. Moreover, $\mathbf{f}(\cdot)$ and the columns of $\mathbf{B}(\cdot)$ are smooth vector fields, $\mathbf{g}_{i}(\cdot)$ is a bounded unknown perturbation term due to parameter variations and external disturbances, and

$$
\operatorname{rank}\left[\mathbf{B}_{i}\left(\mathbf{x}_{1}, \ldots, \mathbf{x}_{i}\right)\right]=n_{i}, \forall \mathbf{x} .
$$

The integers $n_{1}, \ldots, n_{r}$ define the dimension of the $i^{\text {th }}$ block (system structure) and satisfy

$$
n_{1} \leq n_{2} \leq \cdots \leq n_{r}=m, \quad \sum_{i=1}^{r} n_{i}=n .
$$

The control objective is to design a controller such that the output $\mathbf{y}$ in (15) tracks a desired reference $\mathbf{x}_{r e f}(t)$ with bounded derivatives, in spite of unknown but bounded perturbations. To induce quasi sliding mode in the $i^{\text {th }}$ block of the system (15), the continuously differentiable sigmoid function $\operatorname{sigm}(v / \varepsilon)$ defined as

$$
\operatorname{sigm}(v / \varepsilon)=\tanh (v / \varepsilon), \quad \tanh (v / \varepsilon)=\frac{e^{v / \varepsilon}-e^{-v / \varepsilon}}{e^{v / \varepsilon}+e^{-v / \varepsilon}}
$$

where $1 / \mathcal{E}$ is the slope of the sigmoid function at $v=0$, will be used since

$$
\lim _{\varepsilon \rightarrow 0} \operatorname{sigm}(v / \varepsilon)=\operatorname{sign}(v) .
$$

\subsection{Control design}

According to the block control technique (Loukianov, 1998), the state $\mathbf{x}_{i+1}, i=1, \ldots, r-1$ is considered as a virtual control vector in the $i^{\text {th }}$ block of the system (15). The design procedure is described in $r$ steps.

Step 1. The control error in the first block of the system (15) is defined as

$$
\mathbf{z}_{1}=\mathbf{x}_{1}-\mathbf{x}_{r e f}:=\psi_{1}\left(\mathbf{x}_{1}\right)
$$

then

$$
\dot{\mathbf{z}}_{1}=\mathbf{f}_{1}\left(\mathbf{x}_{1}\right)+\mathbf{B}_{1}\left(\mathbf{x}_{1}\right) \mathbf{x}_{2}+\overline{\mathbf{g}}_{1}(t, \mathbf{x})
$$

with $\overline{\mathbf{g}}_{1}(t, \mathbf{x})=\mathbf{g}_{1}(t, \mathbf{x})-\dot{\mathbf{x}}_{r e f}$.

And the virtual control $\mathbf{x}_{2}$ in (16) is redefined of the form

$$
\mathbf{x}_{2}=\mathbf{x}_{2,0}+\mathbf{x}_{2,1}
$$


where the nominal part, $\mathbf{x}_{2,0}$ is selected to eliminate the old dynamics in (16) and introduce the new desired ones, $k_{1} \mathbf{z}_{1}, k_{1}>0$, i. e.

$$
\mathbf{x}_{2,0}=-\mathbf{B}_{1}^{+}\left(\mathbf{x}_{1}\right)\left(\mathbf{f}_{1}\left(\mathbf{x}_{1}\right)+k_{1} \mathbf{z}_{1}-\mathbf{E}_{1} \mathbf{z}_{2}\right), \quad k_{1}>0
$$

where $\mathbf{z}_{2} \in R^{n_{2}}$ is a new variables vector, $\mathbf{E}_{1}=\left[\mathbf{I}_{n_{1}} 0\right] \in R^{n_{1} \times n_{2}}$ and $\mathbf{B}_{1}^{+}$is the right pseudoinverse of $\mathbf{B}_{1}$, defined as $\mathbf{B}_{1}^{+}=\mathbf{B}_{1}^{T}\left(\mathbf{B}_{1} \mathbf{B}_{1}^{T}\right)^{-1}$.

In order to reject the perturbation term $\overline{\mathbf{g}}_{1}(t, \mathbf{x})$ in (16), the second part of the virtual control (17), $\mathbf{x}_{2,1}$ is designed by using the integral sliding mode technique (Utkin et al., 1999). The pseudo-sliding manifold $\mathbf{s}_{1}$ is chosen as

$$
\mathbf{s}_{1}=\mathbf{z}_{1}+\boldsymbol{\sigma}_{1}=0, \quad \mathbf{s}_{1}, \boldsymbol{\sigma}_{1} \in R^{n_{1}} .
$$

Then, from (16)-(19) it follows

$$
\dot{\mathbf{s}}_{1}=-k_{1} \mathbf{z}_{1}+\mathbf{E}_{1} \mathbf{z}_{2}+\mathbf{B}_{1}\left(\mathbf{x}_{1}\right) \mathbf{x}_{2,1}+\overline{\mathbf{g}}_{1}(t, \mathbf{x})+\dot{\boldsymbol{\sigma}}_{1}
$$

Choosing the dynamics for the integral variable $\boldsymbol{\sigma}_{1}$ of the form

$$
\dot{\boldsymbol{\sigma}}_{1}=k_{1} \mathbf{z}_{1}-\mathbf{E}_{1} \mathbf{z}_{2}, \quad \boldsymbol{\sigma}_{1}(0)=-\mathbf{z}_{1}(0)
$$

the equation (20) becomes

$$
\dot{\mathbf{s}}_{1}=\mathbf{B}_{1}\left(\mathbf{x}_{1}\right) \mathbf{x}_{2,1}+\overline{\mathbf{g}}_{1}(t, \mathbf{x}) .
$$

The control input $\mathbf{x}_{2,1}$ in (22) is selected as follows:

$$
\mathbf{x}_{2,1}=-\rho_{1}\left(\mathbf{x}_{1}\right) \mathbf{B}_{1}^{+} \operatorname{sigm}\left(\mathbf{s}_{1} / \varepsilon_{1}\right)
$$

where $\operatorname{sigm}\left(\mathbf{s}_{1} / \varepsilon_{1}\right)=\left[\operatorname{sigm}\left(s_{1,1} / \varepsilon_{1}\right), \ldots, \operatorname{sigm}\left(s_{1, n_{1}} / \varepsilon_{1}\right)\right]^{T}$. Substituting (17), (18) and (23) in (16) results in

$$
\dot{\mathbf{z}}_{1}=-k_{1} \mathbf{z}_{1}+\mathbf{E}_{1} \mathbf{z}_{2}-\rho_{1}\left(\mathbf{x}_{1}\right) \operatorname{sigm}\left(\mathbf{s}_{1} / \varepsilon_{1}\right)+\overline{\mathbf{g}}_{1}(t, \mathbf{x}) .
$$

If the matrix $\mathbf{M}_{1}\left(\mathbf{x}_{1}\right) \in R^{\left(n_{2}-n_{1}\right) \times n_{2}}$ is chosen such that the square matrix

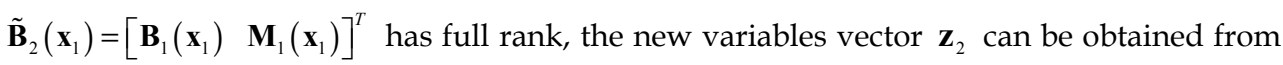
equations (17), (18) and (23) as

$$
\mathbf{z}_{2}=\tilde{\mathbf{B}}_{2} \mathbf{x}_{2}+\left[\begin{array}{c}
\mathbf{f}_{1}\left(\mathbf{x}_{1}\right)-k_{1} \boldsymbol{\psi}_{1}\left(\mathbf{x}_{1}\right)-\rho_{1}\left(\mathbf{x}_{1}\right) \operatorname{sigm}\left(\frac{\mathbf{s}_{1}}{\varepsilon_{1}}\right) \\
0
\end{array}\right]:=\boldsymbol{\psi}_{2}\left(\overline{\mathbf{x}}_{2}\right)
$$


where $\overline{\mathbf{x}}_{2}=\left[\begin{array}{ll}\mathbf{x}_{1} & \mathbf{x}_{2}\end{array}\right]^{T}$. The procedure describe above can be achieved in the $i^{\text {th }}$ block of (15) as follows.

Step i. At this step, the dynamics of the transformed $i^{\text {th }}$ block of the system (15) are given by

$$
\dot{\mathbf{z}}_{i}=\overline{\mathbf{f}}_{i}\left(\overline{\mathbf{x}}_{i}\right)+\overline{\mathbf{B}}_{i}\left(\overline{\mathbf{x}}_{i}\right) \mathbf{x}_{i+1}+\overline{\mathbf{g}}_{i}(t, \mathbf{x})
$$

where $\mathbf{z}_{i} \in R^{n_{i}}$ is a new variables vector, $\overline{\mathbf{g}}_{i}(t, \mathbf{x})=\overline{\mathbf{g}}_{i-1}(t, \mathbf{x})-d / d t\left[\rho_{i-1}\left(\overline{\mathbf{x}}_{i-1}\right) \operatorname{sigm}\left(\mathbf{s}_{i-1} / \varepsilon_{i-1}\right)\right]$, $\mathbf{z}_{i}=\boldsymbol{\psi}_{i}\left(\overline{\mathbf{x}}_{i}\right)$ and $\overline{\mathbf{B}}_{i}=\tilde{\mathbf{B}}_{i} \mathbf{B}_{i}$. The virtual control $\mathbf{x}_{i+1}$ in (26) is redefined as

$$
\mathbf{x}_{i+1}=\mathbf{x}_{i+1,0}+\mathbf{x}_{i+1,1} .
$$

Taking into account the procedure achieved in step $1, \mathbf{x}_{i+1,0}$ and $\mathbf{x}_{i+1,1}$ are selected, respectively, of the form

$$
\begin{gathered}
\mathbf{x}_{i+1,0}=-\overline{\mathbf{B}}_{i}^{+}\left(\overline{\mathbf{x}}_{i}\right)\left(\overline{\mathbf{f}}_{i}\left(\overline{\mathbf{x}}_{i}\right)+k_{i} \mathbf{z}_{i}-\mathbf{E}_{i} \mathbf{z}_{i+1}\right), \quad k_{i}>0 \\
\mathbf{x}_{i+1,1}=-\rho_{i}\left(\overline{\mathbf{x}}_{i}\right) \mathbf{B}_{i}^{+} \operatorname{sigm}\left(\mathbf{s}_{i} / \varepsilon_{i}\right), \quad \rho_{i}>0
\end{gathered}
$$

where $\mathbf{z}_{i+1} \in R^{n_{i+1}}$ is a new variables vector, $\mathbf{E}_{i}=\left[\mathbf{I}_{n_{i}} 0\right] \in R^{n_{i} \times n_{i+1}}$ and $\overline{\mathbf{B}}_{i}^{+}=\overline{\mathbf{B}}_{i}^{T}\left(\overline{\mathbf{B}}_{i} \overline{\mathbf{B}}_{i}^{T}\right)^{-1}$. The proposed pseudo-sliding manifold and its derived dynamics, respectively, are:

$$
\begin{gathered}
\mathbf{s}_{i}=\mathbf{z}_{i}+\boldsymbol{\sigma}_{i}=0, \quad \mathbf{s}_{i}, \boldsymbol{\sigma}_{i} \in R^{n_{i}}, \\
\dot{\mathbf{s}}_{i}=-k_{i} \mathbf{z}_{i}+\mathbf{E}_{i} \mathbf{z}_{i+1}+\overline{\mathbf{B}}_{i}\left(\overline{\mathbf{x}}_{i}\right) \mathbf{x}_{i+1,1}+\overline{\mathbf{g}}_{i}(t, \mathbf{x})+\dot{\boldsymbol{\sigma}}_{i} .
\end{gathered}
$$

If $\boldsymbol{\sigma}_{i}$ satisfies

$$
\dot{\boldsymbol{\sigma}}_{i}=k_{i} \mathbf{z}_{i}-\mathbf{E}_{i} \mathbf{z}_{i+1}, \quad \boldsymbol{\sigma}_{i}(0)=-\mathbf{z}_{i}(0)
$$

the equation (30) can be rewritten as

$$
\dot{\mathbf{s}}_{i}=-\rho_{i}\left(\overline{\mathbf{x}}_{i}\right) \operatorname{sigm}\left(\mathbf{s}_{i} / \varepsilon_{i}\right)+\overline{\mathbf{g}}_{i}(t, \mathbf{x}), \quad \rho_{i}\left(\overline{\mathbf{x}}_{i}\right)>0 .
$$

The substitution of (28) and (29) in the block (26) yields

$$
\dot{\mathbf{z}}_{i}=-k_{i} \mathbf{z}_{i}+\mathbf{E}_{i} \mathbf{z}_{i+1}-\rho_{i}\left(\overline{\mathbf{x}}_{i}\right) \operatorname{sigm}\left(\mathbf{s}_{i} / \varepsilon_{i}\right)+\overline{\mathbf{g}}_{i}(t, \mathbf{x}) .
$$

Again, choosing a $\left(n_{i+1}-n_{i}\right) \times n_{i+1}$ matrix $\mathbf{M}_{i}\left(\overline{\mathbf{z}}_{i}\right)$ such that the square matrix $\tilde{\mathbf{B}}_{i+1}\left(\overline{\mathbf{x}}_{i}\right)=\left[\overline{\mathbf{B}}_{i}\left(\overline{\mathbf{x}}_{i}\right) \mathbf{M}_{i}\left(\overline{\mathbf{x}}_{i}\right)\right]^{T}$ has full rank, the new variables vector $\mathbf{z}_{i+1}$ can be obtained from equations (26)-(29) as

$$
\begin{aligned}
\mathbf{z}_{i+1} & =\tilde{\mathbf{B}}_{i+1} \mathbf{x}_{i+1}+\left[\begin{array}{c}
\left.\overline{\mathbf{f}}_{i}\left(\overline{\mathbf{x}}_{i}\right)-k_{i} \boldsymbol{\psi}_{i}\left(\overline{\mathbf{x}}_{i}\right)-\rho_{i}\left(\overline{\mathbf{x}}_{i}\right) \operatorname{sigm}\left(\frac{\mathbf{s}_{i}}{\varepsilon_{i}}\right)\right], i=2, \ldots, r-1, \\
0
\end{array}\right] \\
& :=\boldsymbol{\Psi}_{i+1}\left(\overline{\mathbf{x}}_{i+1}\right) .
\end{aligned}
$$


Step $r$. At the last step, the transformed complete system can be presented in the new variables $\mathbf{z}_{1}, \ldots, \mathbf{z}_{r}$ as

$$
\begin{aligned}
& \dot{\mathbf{z}}_{i}=-k_{i} \mathbf{z}_{i}+\mathbf{E}_{i} \mathbf{z}_{i+1}-\rho_{i}\left(\overline{\mathbf{x}}_{i}\right) \operatorname{sigm}\left(\mathbf{s}_{i} / \varepsilon_{i}\right)+\overline{\mathbf{g}}_{i}(t, \mathbf{x}) \\
& \dot{\mathbf{s}}_{i}=-\rho_{i}\left(\overline{\mathbf{x}}_{i}\right) \operatorname{sigm}\left(\mathbf{s}_{i} / \varepsilon_{i}\right)+\overline{\mathbf{g}}_{i}(t, \mathbf{x}) \\
& \dot{\mathbf{z}}_{r}=\overline{\mathbf{f}}_{r}(\mathbf{x})+\overline{\mathbf{B}}_{r}(\mathbf{x}) \mathbf{u}+\overline{\mathbf{g}}_{r}(t, \mathbf{x}), i=1, \ldots, r-1
\end{aligned}
$$

where $\overline{\mathbf{B}}_{r}(\cdot)=\tilde{\mathbf{B}}_{r-1}(\cdot) \mathbf{B}_{r}(\cdot)$ has full rank since $n_{r}=m$. Design the control input $\mathbf{u}$ in (32) as

$$
\mathbf{u}=\mathbf{u}_{0}+\mathbf{u}_{1}
$$

and define a sliding variable $\mathbf{s}_{r} \in R^{n_{r}}$ of the form

$$
\mathbf{s}_{r}=\mathbf{z}_{r}+\boldsymbol{\sigma}_{r}, \quad \boldsymbol{\sigma}_{r} \in R^{n_{r}}
$$

Then

$$
\dot{\mathbf{s}}_{r}=\overline{\mathbf{f}}_{r}(\mathbf{x})+\overline{\mathbf{B}}_{r}(\mathbf{x}) \mathbf{u}_{0}+\overline{\mathbf{B}}_{r}(\mathbf{x}) \mathbf{u}_{1}+\overline{\mathbf{g}}_{r}(t, \mathbf{x})+\dot{\boldsymbol{\sigma}}_{r} .
$$

Choosing

$$
\dot{\boldsymbol{\sigma}}_{r}=-\overline{\mathbf{f}}_{r}(\mathbf{x})-\overline{\mathbf{B}}_{r}(\mathbf{x}) \mathbf{u}_{0}, \quad \boldsymbol{\sigma}_{r}(0)=-\mathbf{z}_{r}(0)
$$

simplifies the equation (35) to

$$
\dot{\mathbf{s}}_{r}=\overline{\mathbf{B}}_{r}(\mathbf{x}) \mathbf{u}_{1}+\overline{\mathbf{g}}_{r}(t, \mathbf{x}) .
$$

The second part of the control input (33) is selected as

$$
\mathbf{u}_{1}=-\rho_{r}(\mathbf{x}) \overline{\mathbf{B}}_{r}^{-1} \operatorname{sign}\left(\mathbf{s}_{r}\right), \rho_{r}(\mathbf{x})>0 .
$$

Under the condition $\rho_{r}(\mathbf{x})>\left\|\overline{\mathbf{B}}_{r}^{-1}(\mathbf{x}) \overline{\mathbf{g}}_{r}(t, \mathbf{x})\right\|$ sliding mode occurs on the manifold $\mathbf{s}_{r}=0$ (34) in a finite time. Solving (36) for $\mathbf{u}_{r, 1}$, formally setting $\dot{\mathbf{s}}_{r}=0$, shows

$$
\mathbf{u}_{\mathrm{leq}}=\overline{\mathbf{B}}_{r}^{-1}(\mathbf{x}) \overline{\mathbf{g}}_{r}(t, \mathbf{x})
$$

where $\mathbf{u}_{\text {leq }}(t, \mathbf{x})$ is the equivalent control (Utkin et al., 1999). Therefore, the integral control (37) rejects the perturbation term $\overline{\mathbf{g}}_{r}(t, \mathbf{x})$ in the last block of (32):

$$
\dot{\mathbf{z}}_{r}=\overline{\mathbf{f}}_{r}(\mathbf{x})+\overline{\mathbf{B}}_{r}(\mathbf{x}) \mathbf{u}_{0}+\overline{\mathbf{B}}_{r}(\mathbf{x}) \mathbf{u}_{1 e q}+\overline{\mathbf{g}}_{r}(t, \mathbf{x})
$$

and we have

$$
\dot{\mathbf{z}}_{r}=\overline{\mathbf{f}}_{r}(\mathbf{x})+\overline{\mathbf{B}}_{r}(\mathbf{x}) \mathbf{u}_{0} .
$$

Now, choosing 


$$
\mathbf{u}_{0}=-\overline{\mathbf{B}}_{r}^{-1}(\mathbf{x})\left[\overline{\mathbf{f}}_{r}(\mathbf{x})+k_{r} \mathbf{z}_{r}\right], k_{r}>0
$$

the sliding mode dynamics are described by

$$
\begin{aligned}
& \dot{\mathbf{z}}_{i}=-k_{i} \mathbf{z}_{i}+\mathbf{E}_{i} \mathbf{z}_{i+1}-\rho_{i}\left(\overline{\mathbf{x}}_{i}\right) \operatorname{sigm}\left(\mathbf{s}_{i} / \mathcal{E}_{i}\right)+\overline{\mathbf{g}}_{i}(t, \mathbf{x}) \\
& \dot{\mathbf{s}}_{i}=-\rho_{i}\left(\overline{\mathbf{x}}_{i}\right) \operatorname{sigm}\left(\mathbf{s}_{i} / \mathcal{E}_{i}\right)+\overline{\mathbf{g}}_{i}(t, \mathbf{x}) \\
& \dot{\mathbf{z}}_{r}=-k_{r} \mathbf{z}_{r}, \quad i=1, \ldots, r-1 .
\end{aligned}
$$

Now, it is possible to establish the following result:

Theorem 1. If

H1) the unmatched $\overline{\mathbf{g}}_{1}(\cdot), \ldots, \overline{\mathbf{g}}_{r-1}(\cdot)$ and matched $\overline{\mathbf{g}}_{r}(\cdot)$ perturbations are bounded, i.e., there exist a known scalar function $\beta_{i}(\mathbf{x})$ such that

$$
\left\|\overline{\mathbf{g}}_{i}(t, \mathbf{x})\right\| \leq \beta_{i}(\mathbf{x}), \quad i=1, \ldots, r
$$

then, there exist constants $h_{1}, \ldots, h_{r-1}$ such that the states of the system (38), are uniformly bounded, i.e.

$$
\left\|\mathbf{z}_{i}(t)\right\| \leq h_{i}, i=1, \ldots r-1 .
$$

Moreover the perturbed system (38) reaches to a neighborhood of the output $\mathbf{y}=\mathbf{x}_{1}$ in finite time and remains in this neighborhood.

Proof. The proof is constructive and consists of $r$ steps, begin with the step $r$.

Step $r$. First, the sliding variable $\mathbf{s}_{r}$ stability is analyzed. Considering the Lyapunov function $\mathbf{V}_{r}=\mathbf{s}_{r}{ }^{T} \mathbf{s}_{r}$, it follows:

$$
\dot{\mathbf{V}}_{r}=\mathbf{s}_{r}^{T}\left[-\rho_{r}(\mathbf{x}) \operatorname{sign}\left(\mathbf{s}_{r}\right)+\overline{\mathbf{g}}_{r}(t, \mathbf{x})\right] .
$$

Under the assumption $\mathrm{H1}$, the equation (39) can be written as

$$
\begin{aligned}
\dot{\mathbf{V}}_{r} & =\mathbf{s}_{r}{ }^{T}\left[-\rho_{r}(\mathbf{x}) \operatorname{sign}\left(\mathbf{s}_{r}\right)+\beta_{r}(\mathbf{x})\right] \\
& \leq\left\|\mathbf{s}_{r}\right\|\left[-\rho_{r}(\mathbf{x})+\left\|\beta_{r}(\mathbf{x})\right\|\right] .
\end{aligned}
$$

From (40) it is easy to see that under the condition

$$
\rho_{r}(\mathbf{x})>\left\|\beta_{r}(\mathbf{x})\right\|
$$

the derivative $\dot{\mathbf{V}}_{r}$ is definite negative and the equivalent control $\mathbf{u}_{r, \text { leq }}(t, \mathbf{x})$ satisfies

$$
\mathbf{u}_{r, \text { leq }}=-\overline{\mathbf{g}}_{r}(t, \mathbf{x})
$$

rejecting the perturbation term $\overline{\mathbf{g}}_{r}(t, \mathbf{x})$ in the last block of (38). Now, it is necessary to analyze the stability of the last block. Using the Lyapunov function $\mathbf{V}_{r}=1 / 2 \mathbf{z}_{r}{ }^{T} \mathbf{z}_{r}$, leads to

$$
\dot{\mathbf{V}}_{r} \leq-k_{r}\left\|\mathbf{z}_{r}\right\|^{2}, k_{r}>0 \text {. }
$$


Thus, the trajectories of the last variables vector $\mathbf{z}_{r}$ are asymptotically stable.

Step $r$-1. Proceeding in similar way as in previous step, the Lyapunov function $\mathbf{V}_{r-1}=\mathbf{s}_{r-1}{ }^{T} \mathbf{s}_{r-1}$ is proposed, then

$$
\dot{\mathbf{V}}_{r-1}=\mathbf{s}_{r-1}^{T}\left[-\rho_{r-1}\left(\overline{\mathbf{x}}_{r-1}\right) \operatorname{sigm}\left(\mathbf{s}_{r-1}\right)+\overline{\mathbf{g}}_{r-1}(t, \mathbf{x})\right] .
$$

In the region $\left\|\mathbf{s}_{r-1}\right\|>\varepsilon_{r-1}$ the equation (41) becomes

$$
\begin{aligned}
\dot{\mathbf{V}}_{r-1} & =\mathbf{s}_{r-1}{ }^{T}\left[-\rho_{r-1}\left(\overline{\mathbf{x}}_{r-1}\right) \operatorname{sign}\left(\mathbf{s}_{r-1}\right)+\overline{\mathbf{g}}_{r-1}(t, \mathbf{x})\right] . \\
& \leq\left\|\mathbf{s}_{r-1}\right\|\left[-\rho_{r-1}\left(\overline{\mathbf{x}}_{r-1}\right)+\left\|\overline{\mathbf{g}}_{r-1}(t, \mathbf{x})\right\|\right]
\end{aligned} .
$$

Moreover, under the condition $\rho_{r-1}\left(\overline{\mathbf{x}}_{r-1}\right)>\left\|\overline{\mathbf{g}}_{r-1}(t, \mathbf{z})\right\|,\left\|\mathbf{s}_{r-1}\right\|$ will be decreasing until it reaches the set $\left\{\left\|\mathbf{s}_{r-1}\right\| \leq \varepsilon_{r-1}\right\}$ in a finite time and it remains inside. The upper bound of this reaching time can be calculated by using the comparison lemma (Khalil, 1996) as follows:

$$
t_{r-1} \leq\left\|\mathbf{s}_{r-1}(0)\right\|-\varepsilon_{r-1} .
$$

Furthermore the equivalent control $\mathbf{x}_{r-1,1 \text { leq }}$ fulfills

$$
\dot{\mathbf{s}}_{r-1}=\mathbf{x}_{r-1,1 e q}+\overline{\mathbf{g}}_{r-1}(t, \mathbf{z})=\varepsilon_{r-1} \gamma_{r-1}
$$

where $\varepsilon_{r-1} \gamma_{r-1}$ is the error introduced by using the control law (29). To analyze the stability of the $r$-1 block of the system (38), the Lyapunov function $\mathbf{V}_{r-1}=1 / 2^{\mathbf{z}_{r-1}{ }^{T} \mathbf{z}_{r-1}}$ is considered and its time derivative is given by

$$
\begin{aligned}
\dot{\mathbf{V}}_{r-1} & =\mathbf{z}_{r-1}{ }^{T}\left[-k_{r-1} \mathbf{z}_{r-1}+\mathbf{E}_{r-1} \mathbf{z}_{r}-\rho_{r-1}\left(\overline{\mathbf{x}}_{r-1}\right) \operatorname{sigm}\left(\mathbf{s}_{r-1} / \mathcal{\varepsilon}_{r-1}\right)+\overline{\mathbf{g}}_{r-1}(t, \mathbf{z})\right] \\
& \leq-k_{r-1}\left\|\mathbf{z}_{r-1}\right\|^{2}+\left\|\mathbf{z}_{r-1}\right\|\left[\left\|\mathbf{z}_{r}\right\|-\rho_{r-1}\left(\overline{\mathbf{x}}_{r-1}\right) \operatorname{sigm}\left(\mathbf{s}_{r-1} / \varepsilon_{r-1}\right)+\overline{\mathbf{g}}_{r-1}(t, \mathbf{z})\right] .
\end{aligned}
$$

In the region $\left\|\mathbf{s}_{r-1}\right\|>\varepsilon_{r-1}$, the derivative $\dot{\mathbf{V}}_{r-1}$ becomes

$$
\begin{aligned}
\dot{\mathbf{V}}_{r-1} & \leq-k_{r-1}\left\|\mathbf{z}_{r-1}\right\|^{2}+\left\|\mathbf{z}_{r-1}\right\|\left[\left\|\mathbf{z}_{r}\right\|-\rho_{r-1} \operatorname{sign}\left(\mathbf{s}_{r-1} / \mathcal{\varepsilon}_{r-1}\right)+\overline{\mathbf{g}}_{r-1}(t, \mathbf{z})\right] \\
& \leq-k_{r-1}\left\|\mathbf{z}_{r-1}\right\|^{2}+\left\|\mathbf{z}_{r-1}\right\|\left[\left\|\mathbf{z}_{r}\right\|+\dot{\mathbf{s}}_{r-1}\right]
\end{aligned}
$$

and considering (43), it can be rewritten as

$$
\dot{\mathbf{V}}_{r-1} \leq-k_{r-1}\left\|\mathbf{z}_{r-1}\right\|^{2}+\left\|\mathbf{z}_{r-1}\right\|\left[\left\|\mathbf{z}_{r}\right\|+\varepsilon_{r-1} \boldsymbol{\gamma}_{r-1}\right] .
$$

Suppose that $\varepsilon_{r-1} \gamma_{r-1}$ satisfies the following bound:

$$
\varepsilon_{r-1} \gamma_{r-1} \leq \alpha_{r-1}\left\|\mathbf{z}_{r-1}\right\|+\beta_{r-1}, \alpha_{r-1}, \beta_{r-1} \in R .
$$

Then it is possible to present the equation (44) of the form 


$$
\begin{aligned}
\dot{\mathbf{V}}_{r-1} & \leq-k_{r-1}\left\|\mathbf{z}_{r-1}\right\|^{2}+\left\|\mathbf{z}_{r-1}\right\|\left[\left\|\mathbf{z}_{r}\right\|+\alpha_{r-1}\left\|\mathbf{z}_{r-1}\right\|+\beta_{r-1}\right] \\
& \leq-\left\|\mathbf{z}_{r-1}\right\|\left[\left(k_{r-1}-\alpha_{r-1}\right)\left\|\mathbf{z}_{r-1}\right\|-\left\|\mathbf{z}_{r}\right\|-\beta_{r-1}\right]
\end{aligned}
$$

which is negative in the region

$$
\left\|\mathbf{z}_{r-1}\right\|>\delta_{r-1}\left\|\mathbf{z}_{r}\right\|+\lambda_{r-1}
$$

where $\delta_{r-1}=\frac{1}{k_{r-1}-\alpha_{r-1}}$ and $\lambda_{r-1}=\frac{\beta_{r-1}}{k_{r-1}-\alpha_{r-1}}$. Moreover $\delta_{r-1}$ and $\lambda_{r-1}$ are positive for $k_{r-1}>\alpha_{r-1}$. Thus the trajectories of the vector state enter ultimately in the region defined by

$$
\left\|\mathbf{z}_{r-1}\right\| \leq \delta_{r-1}\left\|\mathbf{z}_{r}\right\|+\lambda_{r-1} .
$$

Step $i$. The step $r-1$ can be generalized for the block $i$, with $i=r-1, r-2, \ldots, 1$.

In the region $\left\|\mathbf{s}_{i}\right\|>\varepsilon_{i}$ the derivative of the Lyapunov function $\mathbf{V}_{i}=\mathbf{s}_{i}^{T} \mathbf{s}_{i}$, is calculated as

$$
\begin{aligned}
\dot{\mathbf{V}}_{i} & =\mathbf{s}_{i}{ }^{T}\left[-\rho_{i}\left(\overline{\mathbf{x}}_{i}\right) \operatorname{sign}\left(\mathbf{s}_{i}\right)+\overline{\mathbf{g}}_{i}(t, \mathbf{x})\right] \\
& \leq\left\|\mathbf{s}_{i}\right\|\left[-\rho_{i}\left(\overline{\mathbf{x}}_{i}\right)+\left\|\overline{\mathbf{g}}_{i}(t, \mathbf{x})\right\|\right] .
\end{aligned}
$$

Again, under the condition $\rho_{i}\left(\overline{\mathbf{x}}_{i}\right)>\left\|\overline{\mathbf{g}}_{i}(t, \mathbf{z})\right\|, \mathbf{s}_{i}$ enter in the region $\left\{\left\|\mathbf{s}_{i}\right\| \leq \varepsilon_{i}\right\}$ in a finite time given by

$$
t_{i} \leq\left\|\mathbf{s}_{i}(0)\right\|-\varepsilon_{i}
$$

The equivalent control $\mathbf{x}_{i, \text { leq }}$ satisfies

$$
\dot{\mathbf{s}}_{i}=\mathbf{x}_{i, 1 \text { leq }}+\overline{\mathbf{g}}_{i}(t, \mathbf{z})=\varepsilon_{i} \gamma_{i} .
$$

Considering the function $\mathbf{V}_{i}=1 / 2 \mathbf{z}_{i}^{T} \mathbf{z}_{i}$ inside the subspace $\left\|\mathbf{s}_{i}\right\|>\varepsilon_{i}$, it follows

$$
\begin{aligned}
\dot{\mathbf{V}}_{i} & \leq-k_{i}\left\|\mathbf{z}_{i}\right\|^{2}+\left\|\mathbf{z}_{i}\right\|\left[\left\|\mathbf{z}_{i+1}\right\|-\rho_{i}\left(\overline{\mathbf{x}}_{i}\right) \operatorname{sign}\left(\mathbf{s}_{i} / \varepsilon_{i}\right)+\overline{\mathbf{g}}_{i}(t, \mathbf{z})\right] \\
& \leq-k_{i}\left\|\mathbf{z}_{i}\right\|^{2}+\left\|\mathbf{z}_{i}\right\|\left[\left\|\mathbf{z}_{i+1}\right\|+\dot{\mathbf{s}}_{i}\right]
\end{aligned}
$$

and with (47), $\dot{\mathbf{V}}_{i}$ becomes

$$
\dot{\mathbf{V}}_{i} \leq-k_{i}\left\|\mathbf{z}_{i}\right\|^{2}+\left\|\mathbf{z}_{i}\right\|\left[\left\|\mathbf{z}_{i+1}\right\|+\varepsilon_{i} \gamma_{i}\right]
$$

Supposing that $\varepsilon_{i} \gamma_{i}$ fulfills

$$
\varepsilon_{i} \gamma_{i} \leq \alpha_{i}\left\|\mathbf{z}_{i}\right\|+\beta_{i}, \alpha_{i}, \beta_{i} \in R
$$

then

$$
\dot{\mathbf{V}}_{i} \leq-\left\|\mathbf{z}_{i}\right\|\left[\left(k_{i}-\alpha_{i}\right)\left\|\mathbf{z}_{i}\right\|-\left\|\mathbf{z}_{i+1}\right\|-\beta_{i}\right]
$$

which is negative in the region 


$$
\left\|\mathbf{z}_{i}\right\|>\delta_{i}\left\|\mathbf{z}_{i+1}\right\|+\lambda_{i}
$$

where $\delta_{i}=\frac{1}{k_{i}-\alpha_{i}}$ and $\lambda_{i}=\frac{\beta_{i}}{k_{i}-\alpha_{i}}$, which are positive for $k_{i}>\alpha_{i}$. Therefore a solution for $\mathbf{z}_{i}$ is ultimately bounded by

$$
\left\|\mathbf{z}_{i}\right\| \leq \delta_{i}\left\|\mathbf{z}_{i+1}\right\|+\lambda_{i}
$$

Then with the bound

$$
\varepsilon_{i} \gamma_{i} \leq \alpha_{i}\left\|\mathbf{z}_{i}\right\|+\beta_{i}, i=1,2, \ldots, r-1
$$

the convergence region is defined by:

$$
\begin{aligned}
& \left\|\mathbf{z}_{r-1}\right\|>\delta_{r-1}\left\|\mathbf{z}_{r}\right\|+\lambda_{r-1}:=h_{r-1} \\
& \left\|\mathbf{z}_{r-2}\right\|>\delta_{r-2}\left\|\mathbf{z}_{r-1}\right\|+\lambda_{r-2}:=h_{r-2} \\
& \vdots \\
& \left\|\mathbf{z}_{1}\right\|>\delta_{1}\left\|\mathbf{z}_{2}\right\|+\lambda_{1}:=h_{1} .
\end{aligned}
$$

\section{PES control design}

Since the subsystem (10) has the NBC form, the ISM technique will be applied to design a robust controller for EPS. First, the rotor speed stability will be achieved. Secondly, the terminal voltage generator controller is outlined. Then, a switching logic is proposed to coordinate the operation of both controllers. Finally, an EPS observer is introduced.

\subsection{Integral Sliding Mode Speed Stabilizer (ISMSS)}

To achieve the first control objective, that is, the rotor speed stability enhancement, define the control error as (Huerta-Avila et al., 2007a, Huerta-Avila et al., 2007b)

$$
z_{2 i}=x_{2 i}-\omega_{b} .
$$

Taking the time derivative of (48) along the trajectories of (10) yields

$$
\dot{z}_{2 i}=f_{\omega i}\left(\mathbf{x}_{i}, \mathbf{v}_{i}\right)-q_{i}\left(\mathbf{x}_{i}, \mathbf{v}_{i}\right) x_{3 i}+g_{2 i}\left(\mathbf{x}_{i}, \mathbf{v}_{i}, T_{m i}\right)
$$

where $\mathbf{x}_{i}=\left(\mathbf{x}_{1 i} \mathbf{x}_{2 i}\right)^{T}, q_{i}(t)>0, \forall t>0$.

Redefine the virtual control, $x_{3 i}$ in (49) as

$$
x_{3 i}=x_{3 i, 0}+x_{3 i, 1} .
$$

The desired dynamics for $z_{2 i}$ is chosen of the form

$$
\dot{z}_{2 i}=-k_{i} z_{2 i}+z_{3 i}+q_{i}\left(\mathbf{x}_{i}, \mathbf{v}_{i}\right) x_{3 i, 1}+g_{2 i}\left(\mathbf{x}_{i}, \mathbf{v}_{i}, T_{m i}\right), k_{i}>0
$$

These dynamics can be obtained by choosing $x_{3 i, 0}$ as 


$$
x_{3 i, 0}=-\left[q_{i}\left(\mathbf{x}_{i}, \mathbf{v}_{i}\right)\right]^{-1}\left[f_{\omega i}\left(\mathbf{x}_{i}, \mathbf{v}_{i}\right)+k_{i} z_{2 i}-z_{3 i}\right]
$$

where $z_{3 i}$ is a new variable. To design the second part of (50), $x_{3 i, 1}$, define a pseudo-sliding variable $s_{2 i}$ as

$$
s_{2 i}=z_{2 i}+\sigma_{2 i}
$$

with the integral variable $\sigma_{2 i}$. Using (49)-(51), it follows

$$
\dot{S}_{2 i}=-k_{0 i} z_{2 i}+z_{3 i}+q_{i}\left(\mathbf{x}_{i}, \mathbf{v}_{i}\right) x_{3 i, 1}+g_{2 i}\left(\mathbf{x}_{i}, \mathbf{v}_{i}, T_{m i}\right)+\dot{\sigma}_{2 i}
$$

Choosing

$$
\dot{\sigma}_{2 i}=k_{2 i} z_{2 i}-z_{3 i}, \quad \sigma_{2 i}(0)=-z_{2 i}(0)
$$

the equation (53) becomes

$$
\dot{S}_{2 i}=g_{2 i}\left(\mathbf{x}_{i}, \mathbf{v}_{i}, T_{m i}\right)+q_{i}\left(\mathbf{x}_{i}, \mathbf{v}_{i}\right) x_{3 i, 1} .
$$

Select $x_{3 i, 1}$ of the form

$$
x_{3 i, 1}=-\rho_{2 i} \operatorname{sigm}\left(s_{2 i} / \varepsilon_{i}\right), \rho_{2 i}>0 .
$$

Then, the sliding variable $s_{\omega i}=z_{3 i}$ is defined from (50), (52) and (54) of the form

$$
s_{\omega i}=f_{\omega i}\left(\mathbf{x}_{i}, \mathbf{v}_{i}\right)+q_{i}\left(\mathbf{x}_{i}, \mathbf{v}_{i}\right) x_{3 i}+k_{0 i} z_{2 i}+\rho_{2 i} \operatorname{sigm}\left(s_{2 i} / \varepsilon_{i}\right) .
$$

Thus, straightforward algebra reveals

$$
\dot{s}_{\omega i}=f_{s i}\left(\mathbf{x}_{i}, \mathbf{v}_{i}\right)+b_{s i}\left(\mathbf{x}_{i}, \mathbf{v}_{i}\right) v_{f i}
$$

where $f_{s i}(\cdot)$ is a continuous function and $b_{s i}(\cdot)=q_{i}(\cdot) b_{i 4}$.

Considering (56), under the condition

$$
k_{g i}>\left|b_{s i}^{-1}\left(\mathbf{x}_{i}, \mathbf{v}_{i}\right) f_{s i}\left(\mathbf{x}_{i}, \mathbf{v}_{i}\right)\right|
$$

the proposed discontinuous control law

$$
v_{f i}=-k_{g i} \operatorname{sign}\left(s_{\omega i}\right), \quad k_{g i}>0
$$

ensures the convergence of the state to the manifold $s_{\omega i}=z_{3 i}=0$ (55) in a finite time (Utkin et al., 1999). The sliding mode motion on this manifold is governed by the reduced order system

$$
\begin{aligned}
& \dot{x}_{1 i}=z_{2 i}, \\
& \dot{z}_{2 i}=-k_{0 i} z_{2 i}-\rho_{2 i} \operatorname{sigm}\left(s_{2 i} / \varepsilon_{i}\right)+g_{2 i}\left(\mathbf{x}_{i}, \mathbf{v}_{i}, T_{m i}\right) \\
& \dot{s}_{2 i}=-\rho_{2 i} \operatorname{sigm}\left(s_{2 i} / \varepsilon_{i}\right)+g_{2 i}\left(\mathbf{x}_{i}, \mathbf{v}_{i}, T_{m i}\right)
\end{aligned}
$$




$$
\dot{\mathbf{x}}_{2 i}=\mathbf{A}_{2 i} \mathbf{x}_{2 i}+\tilde{\mathbf{f}}_{2 i}\left(\mathbf{x}_{i}, \mathbf{v}_{i}\right)
$$

Now, choosing $\varepsilon_{i}$ be sufficiently small and under the condition

$$
\rho_{2 i}>\left|g_{2 i}\left(\mathbf{x}_{i}, \mathbf{v}_{i}, T_{m i}\right)\right|
$$

a quasi sliding mode motion is enforced in a small $\varepsilon_{i}$-vicinity of $s_{2 i}=0$. Thus, if $\varepsilon_{i} \rightarrow 0$ then the perturbation term $g_{2 i}\left(\mathbf{x}_{i}, \mathbf{v}_{i}, T_{m i}\right)$ in (59) is rejected, and the linearized mechanical dynamics can be represented as

$$
\begin{aligned}
& \dot{x}_{1 i}=z_{2 i} \\
& \dot{z}_{2 i}=-k_{0 i} z_{2 i}
\end{aligned}
$$

with the desired eigenvalue $-k_{0 i}$.

The equation (59) represents the rotor flux internal dynamics. The matrix $\mathbf{A}_{2 i}$ is Hurwitz and the nonvanishing perturbation $\tilde{\mathbf{f}}_{2 i}\left(\mathbf{x}_{i}, \mathbf{V}_{i}\right)$ is a continuous function. Therefore there exists an admissible region where a solution $\mathbf{x}_{2 i}(t)$ of (60) is ultimately bounded (Khalil, 1996). Moreover, the control error $z_{2 i}$ (48) tends exponentially to zero, and the angle $x_{1 i}$ tends to a constant steady state, $\delta_{s s i}$.

Remark: Since the initial conditions of the EPS are availabe, it is possible to apply the integral sliding modes technique.

\subsection{Sliding Mode Voltage Regulator}

In this subsection, the voltage regulation problem is studied. The terminal voltage, $v_{g i}$, is defined as

$$
v_{g i}^{2}=v_{d i}^{2}+v_{q i}^{2} .
$$

Using (8), $v_{d i}$ and $v_{q i}$ are calculated of the form

$$
\mathbf{v}_{i}=\left[\begin{array}{c}
v_{d i} \\
v_{q i}
\end{array}\right]=-\mathbf{H}_{i}^{-1}\left[\mathbf{A}_{z i} \mathbf{i}_{i}+\mathbf{f}_{z i}\left(\mathbf{x}_{i}\right)\right] .
$$

Then, the dynamics for terminal voltage, $v_{g i}$ can be obtained from (61), (62), (6), and (7) as (Loukianov, et al., 2006)

$$
\dot{v}_{g i}=f_{v i}\left(\mathbf{x}_{i}, \mathbf{i}_{i}\right)+b_{v i} v_{f i}+g_{v i}\left(\mathbf{x}_{i}, \mathbf{i}_{i}, T_{m i}\right)
$$

where $f_{v i}\left(\mathbf{x}_{i}, \mathbf{i}_{i}\right)$ is the nominal part of the voltage dynamics and the perturbation term $g_{v i}\left(\mathbf{x}_{i}, \mathbf{i}_{i}, T_{m i}\right)$ contains parameter variations and external disturbances, $b_{v i}=h_{i 2} b_{i 4}$, $b_{v i}(t), \forall t \geq 0$. For the details see Appendix.

Defining the voltage control error 


$$
e_{v i}=v_{g i}-v_{r e f i}
$$

and the control input $v_{f i}$

$$
v_{f i}=v_{f i, 0}+v_{f i, 1}
$$

we have

$$
\dot{e}_{v i}=f_{v i}\left(\mathbf{x}_{i}, \mathbf{i}_{i}\right)+b_{v i} v_{f i, 0}+b_{v i} v_{f i, 1}+g_{v i}\left(\mathbf{x}_{i}, \mathbf{i}_{i}, T_{m i}\right)
$$

where $v_{\text {refi }}$ is the constant reference voltage. To design a robust controller we use the integral sliding mode approach (Utkin et al., 1999). In order to reject the perturbation term $g_{v i}\left(\mathbf{x}_{i}, \mathbf{i}_{i}, T_{m i}\right)$ in (65) a sliding variable $s_{v i} \in R$ is formulated as

$$
S_{v i}=e_{v i}+\sigma_{v i}
$$

with the integral variable $\sigma_{v i} \in R$. Then from (65) and (66) it follows

$$
\dot{s}_{v i}=f_{v i}\left(\mathbf{x}_{i}, \mathbf{i}_{i}\right)+b_{v i} v_{f i, 0}+b_{v i} v_{f i, 1}+g_{v i}\left(\mathbf{x}_{i}, \mathbf{i}_{i}, T_{m i}\right)+\dot{\sigma}_{v i}
$$

Choosing

$$
\dot{\sigma}_{v i}=-f_{v i}\left(\mathbf{x}_{i}, \mathbf{i}_{i}\right)-b_{v i} v_{f i, 0}, \sigma_{v i}(0)=-e_{v i}(0)
$$

results in

$$
\dot{s}_{v i}=b_{v i} v_{f i, 1}+g_{v i}\left(\mathbf{x}_{i}, \mathbf{i}_{i}, T_{m i}\right)
$$

Select $v_{f i, 1}$ in (68) as

$$
v_{f i, 1}=-\rho_{2 i} \operatorname{sign}\left(s_{v i}\right), \rho_{2 i}>0 .
$$

From (68), under the condition $\rho_{2 i}>\left|b_{v i}{ }^{-1} g_{v i}\left(\mathbf{x}_{i}, \mathbf{i}_{i}, T_{m i}\right)\right|$ a sliding mode is enforced on the manifold $s_{v i}=0$ (66) from the initial time instant $t=0$. The equivalent control

$$
v_{f i, l e q}=-b_{v i}^{-1} g_{v i}\left(\mathbf{x}_{i}, \mathbf{i}_{i}, T_{m i}\right)
$$

calculated as a solution of $\dot{s}_{v i}=0$ (67), compensates exactly the perturbation term $g_{v i}\left(\mathbf{x}_{i}, \mathbf{i}_{i}, T_{m i}\right)$ in (63) (Utkin et al., 1999), and the sliding mode motion is described by the unperturbed system

$$
\dot{e}_{v i}=f_{v i}\left(\mathbf{x}_{i}, \mathbf{i}_{i}\right)+b_{v i} v_{f i, 0} .
$$

Now, it is necessary to achieve the terminal voltage regulation, i. e. the control input $v_{f i, 0}$ in (70) is selected of the form

$$
v_{f i, 0}=-k_{g} \operatorname{sign}\left(e_{v i}\right)
$$

From (70) and (71), we have 


$$
\dot{e}_{v i}=f_{v i}\left(\mathbf{x}_{i}, \mathbf{i}_{i}\right)-k_{g} b_{v i} \operatorname{sign}\left(e_{v i}\right) .
$$

Then, under the condition

$$
k_{g}>\left|b_{v i}^{-1} f_{v i}\left(\mathbf{x}_{i}, \mathbf{i}_{i}\right)\right|
$$

the terminal voltage control error $e_{v i}$ tends to zero in a finite time (Utkin et al., 1999).

\subsection{Control logic}

There are two control objectives: the rotor speed stabilization and the terminal voltage regulation for each generator in the EPS. However, only one control input is available, the excitation voltage $v_{f i}$. Then, the following control logic is proposed:

$$
v_{f i}=\left\{\begin{array}{lll}
-k_{g i} \operatorname{sign}\left(s_{\omega i}\right), & \text { if } & \left|s_{\omega i}\right|>\beta_{i} \\
-k_{g} \operatorname{sign}\left(e_{v i}\right)-\rho_{2 i} \operatorname{sign}\left(s_{v i}\right), & \text { if } & \left|s_{\omega i}\right| \leq \beta_{i}
\end{array}, \beta_{i}=\left\{\begin{array}{lll}
\beta_{1 i} \text { if } & \left|\beta_{v i}\right|>\beta_{3 i} \\
\beta_{2 i} \text { if } & \left|\beta_{v i}\right| \leq \beta_{3 i}
\end{array}\right.\right.
$$

with $\beta_{2 i}<\beta_{1 i}$. Basically, a hierarchical control action through the proposed logic (74) is presented. First, the mechanical dynamics is stabilized by means of the ISMSS, yielding the stabilization of the speed switching manifold $s_{\omega i}$. When $s_{\omega i}$ reaches to a region defined by $\beta_{1 i}$, the control resources are dedicated to stabilize the terminal voltage error $\beta_{v i}$. After the convergence of $\beta_{v i}$ such that $\left|\beta_{v i}\right| \leq \beta_{3 i}$, the control logic reduces the $s_{\omega i}$ boundary layer width from $\beta_{1 i}$ to $\beta_{2 i}$. Thus, the controller maintains the value of $s_{\omega i}$ within desired accuracy $\left|s_{\omega i}\right| \leq \beta_{2 i}$ and $\left|e_{v i}\right| \leq \beta_{3 i}$. Figure 1 shows the schematic diagram of the proposed controller.

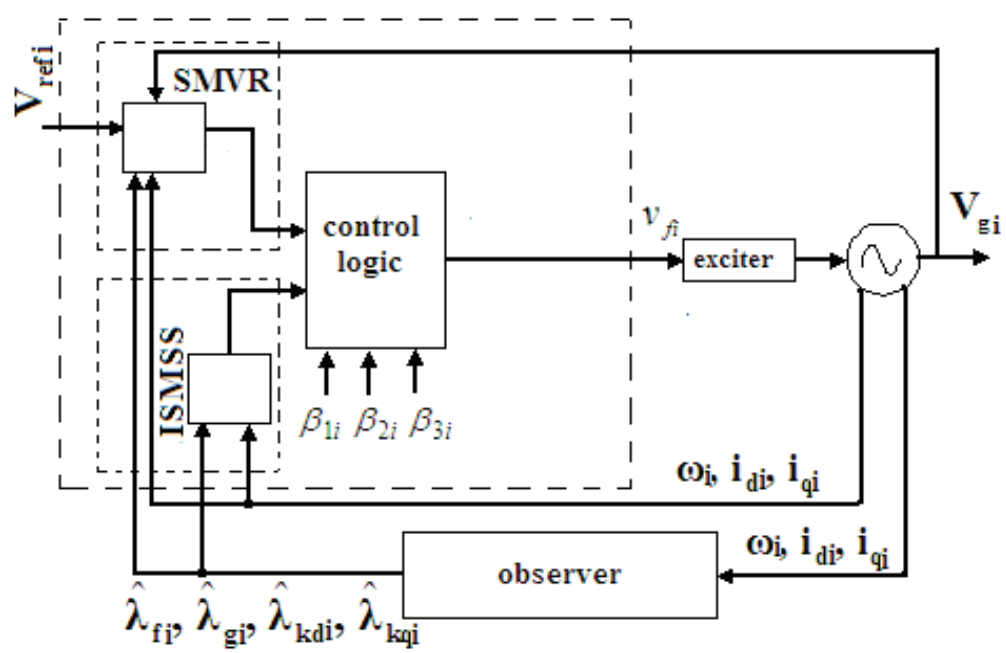

Figure 1. Proposed controller schematic diagram 


\subsection{EPS observer}

Since the control scheme (74) needs the values of the rotor fluxes, it is neccesary to design a observer for the EPS. Assume that the power angle, $x_{1 i}$, rotor speed, $x_{2 i}$ and stator currents $i_{d i}$ and $i_{q i}$ can be measured.

The rotor fluxes $x_{3 i}, x_{4 i}, x_{5 i}$ and $x_{6 i}$ can be estimated by means of the following observer:

$$
\left[\begin{array}{l}
\dot{\hat{x}}_{3 i} \\
\dot{\hat{x}}_{4 i} \\
\dot{\hat{x}}_{5 i} \\
\dot{\hat{x}}_{6 i}
\end{array}\right]=\left[\begin{array}{c}
b_{i 1} \hat{x}_{3 i}+b_{i 2} \hat{x}_{5 i}+b_{i 3} i_{d i} \\
c_{i 1} \hat{x}_{4 i}+c_{i 2} \hat{x}_{6 i}+c_{i 3} i_{q i} \\
d_{i 1} \hat{x}_{3 i}+d_{i 2} \hat{x}_{5 i}+d_{i 3} i_{d i} \\
r_{i 1} \hat{x}_{4 i}+r_{i 2} \hat{x}_{6 i}+r_{i 3} i_{q i}
\end{array}\right]+\left[\begin{array}{c}
b_{i 4} \\
0 \\
0 \\
0
\end{array}\right] v_{f i}
$$

where $\hat{\mathbf{x}}_{i}=\left[\hat{x}_{3 i}, \hat{x}_{4 i}, \hat{x}_{5 i}, \hat{x}_{6 i}\right]^{T}$ are the estimate of the rotor fluxes. The convergence of the observer (75) can be analyzed by the error dynamics obtained from (75) and (6), given by the linear system:

$$
\dot{\mathbf{e}}_{i}=\mathbf{A}_{0 i}
$$

with $\mathbf{e}_{i}=\left[e_{3 i}, \ldots, e_{6 i}\right], e_{j i}=x_{j i}-\hat{x}_{j i}, j=3, \ldots, 6, \mathbf{A}_{0 i}=\left[\begin{array}{cccc}b_{i 1} & 0 & b_{i 2} & 0 \\ 0 & c_{i 1} & 0 & c_{i 2} \\ d_{i 1} & 0 & d_{i 2} & 0 \\ 0 & r_{4 i} & 0 & r_{i 2}\end{array}\right]$.

The eigenvalues of the matrix $\mathbf{A}_{0 i}$ calculated as

$$
\begin{aligned}
& p_{1,2 i}=\frac{1}{2}\left(c_{i 1}+r_{i 2}\right) \pm \frac{1}{2} \sqrt{c_{i 1}^{2}+r_{i 2}{ }^{2}-2 c_{i 1} r_{i 2}+4 c_{i 2} r_{i 1}}, \\
& p_{3,4 i}=\frac{1}{2}\left(b_{i 1}+d_{i 2}\right) \pm \frac{1}{2} \sqrt{b_{i 1}{ }^{2}+d_{i 2}{ }^{2}-2 b_{i 1} d_{i 2}+4 b_{i 2} d_{i 1}}
\end{aligned}
$$

are real and negative. Therefore, the solution of the subsystem (76) is exponentially stable. The resulting estimates rotor fluxes are employed in the control logic (74) instead of the real variables.

\section{Simulations results}

The proposed control algorithm was tested on the equivalent model of the WSCC, (Western System Coordinating Council, Nine buses, three generators, three loads), fig. 2, (Anderson \& Fouad, 1994). The parameters of the generators and network used in the simulation were taken from (Anderson \& Fouad, 1994) (see Appendix).

Figures 3-8 depict results under four different events:

a. at $t=1 \mathrm{~s}$, experienced a pulse $0.5 \mathrm{p}$.u. for $1 \mathrm{~s}$ in the generator 2 ,

b. at $t=4 \mathrm{~s}$ until $t=4.15 \mathrm{~s}$, a three-phase short circuit is simulated in the terminals of generator 1 ,

c. at $t=10 \mathrm{~s}$, a three-phase short circuit during $150 \mathrm{~ms}$ is applied in the line 5-7 (see fig. 2); the fault is cleared by opening the line, and 
d. at $t=15 \mathrm{~s}$, it was introduced a parametric variations, by incrementing up to $25 \%$ the parameters $L_{m i}$ in the generators.

Figures 3 and 5 show the relative angles and speed response of the close-loop system, respectively with a type I excitation system with PSS (Anderson \& Fouad, 1994, EPRI, 1977). Figure 8 show the proposed observer converge in spite of perturbations.

Figures 4-7 reveal some important aspects:

1. The state variables fastly reach a steady state condition after small and large disturbances, showing the robust stability of the closed-loop system.

2. The controller is able to improve both, the power system stabilization and the post-fault terminal voltage regulation.

Comparing the transient speed response of the generators in case of ISMSS /SMVR and AVR/ PSS controllers shown in Figures 6 and 5 respectively, we have some important observations:

1. The traditional AVR/PSS stabilizes the system. However, the transient response of the classical controller is more oscillatory than the response given by the proposed nonlinear ISMSS /SMVR one since the latter adds significantly better damping in the power oscillations. It is possible to observe that the overshoot and settling time are reduced as well.

2. The performance of the ISMSS /SMVR is robust under different operating conditions. Figures 4 and 6 show clearly that the robustness of the controller under generators parameters variations and changes on the network configuration, such as disconnection of lines and incrementing and / or decrementing of loads. Thus the performance of the proposed ISMSS /SMVR controller tends to be unaffected.

3. Since the ISMSS /SMVR adds additional damping, the transient response controller is better compared to other ones (see for instance (Ahmed at al., 1996)). With the ISMSS /SMVR, the settling time is lesser and the overshot is shorter than the shown by the suboptimal robust controller presented in (Ahmed at al., 1996).

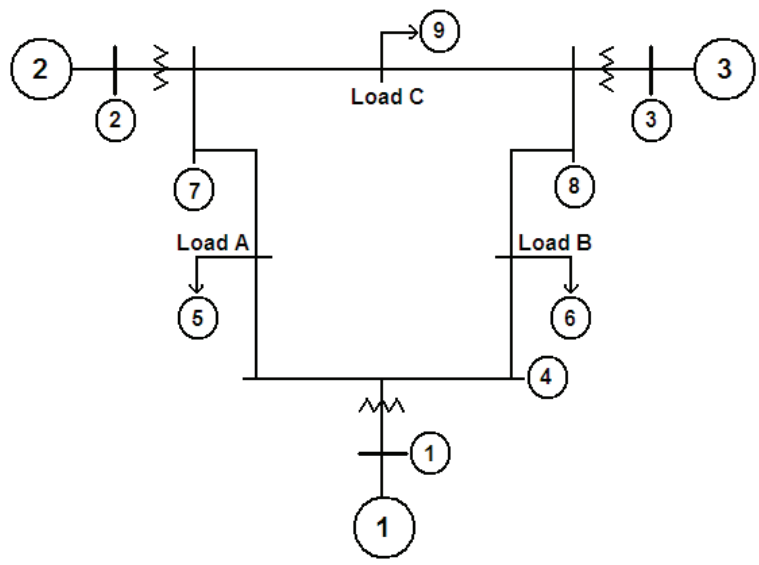

Figure 2. WSCC diagram 


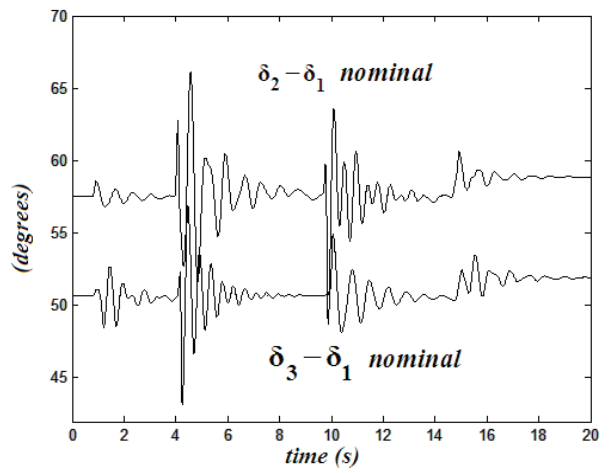

Figure 3. Relative angles response with classical control

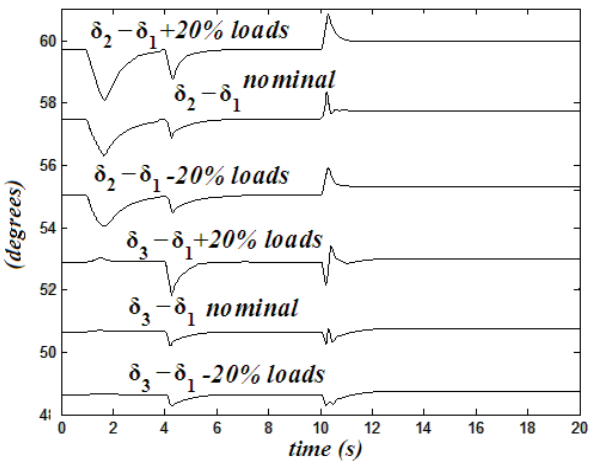

Figure 4. Relative angles response with the proposed controller
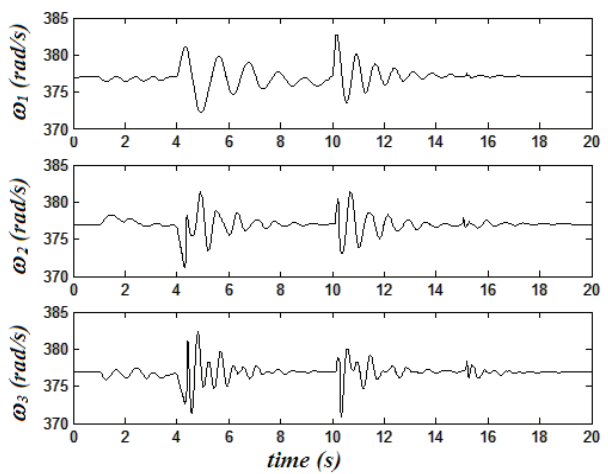

Figure 5. Speed of the three generators response with classical control 

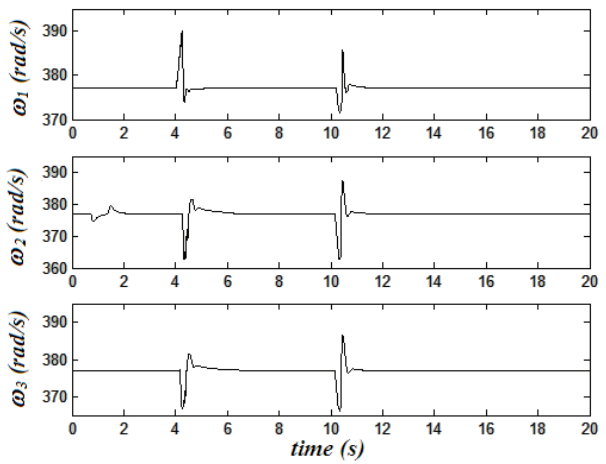

Figure 6. Speed of the three generators response with the proposed controller
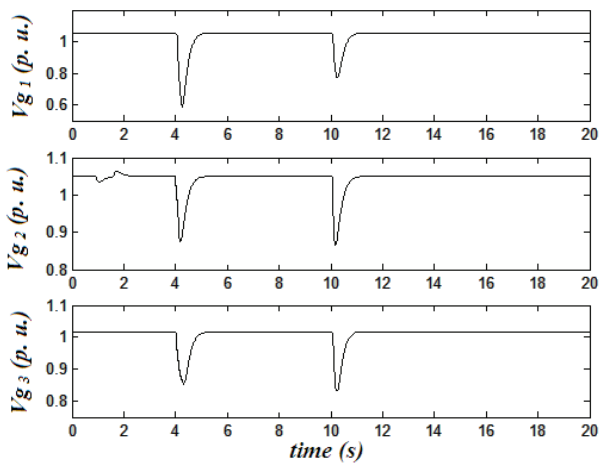

Figure 7. Terminal voltage of the three generators response with the proposed controller
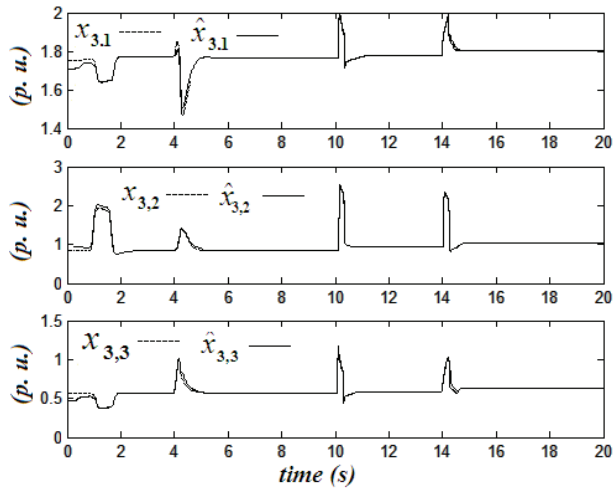

Figure 8. Field flux of the three generators response 


\section{Conclusions}

The ISM with block control technique as a novel nonlinear control technique for the class of nonlinear systems presented in the NBC form was presented. The control methodology was explained step-by-step, and the stability conditions were found for each step. The ISM technique is robust under unknown but bounded matched and/or unmatched perturbations.

Then, in order to test the effectiveness of the ISM technique, a controller for EPS was designed. A plant model used for control is fully detailed nonlinear, and this model takes into account all interactions in power system between the electrical and mechanical dynamics and load constraints. With the proposed control scheme, the only local information is required. The stability analysis of the closed-loop EPS controller, including an observer was carried out. The designed ISMSS/SMVR was tested through simulation under the most important perturbations in the EPS:

1. Variation of the mechanical torque.

2. Large fault (a $150 \mathrm{~ms}$ short circuit).

3. Loads variations.

4. Generator parameter variations.

The simulation results show that the sliding mode controller with the proposed logic is able to achieve the mechanical dynamics and the generator terminal voltages robust stability under small and large disturbances.

The proposed performance of the nonlinear ISMSS/ SMVR control system (74) is independent from the operating point of the system. It is important to note that the proposed nonlinear control scheme ensures cancellation of the interactions between the subsystems provided an additional damping with respect to classical controllers.

\section{References}

Abidi, K. \& Šabanovic, A., (2007). Sliding-mode control for high-precision motion of a piezostage, IEEE Trans. on Industrial Electronics, vol. 54, no. 1, pp. 629-637, 2007.

Adhami-Mirhosseini, A. \& Yazdanpanah, M. J., (2005). Robust Tracking of perturbed systems by nested sliding mode control. Proc. of ICCA2005, Budapest, Hungary, June 2005.

Aggoune, M. E., Boudjeman, F., Bensenouci, A., Hellal, A., Elmesai, M.R., \& Vadari, S.V., (1994). Design of Variable Structure Voltage Regulator Using Pole Assignment Technique, IEEE Transaction on Automatic Control, Vol. 39, No. 10, October 1994.

Ahmed, S. S., Chen, L. and Petroianu, A., (1996), Design of Suboptimal Hळ Excitation Controller, IEEE Trans. on Power Systems, Vol. 11, No. 1, February, 1996.

Akhkrif, O., Okou, F., Dessaint, L., \& Champagne, R., (1999). Application of Multivariable Feedback Linearization Scheme for Rotor Angle Stability and Voltage Regulation of Power System, IEEE Trans. Power Syst., Vol.14, No.2, pp.620-628, 1999.

Anderson, P. M., \& Fouad, A., (1994). Power System Control and Stability, IEEE Press New York, 1994.

Bandal, V., Bandyopadhyay, B., \& Kulkarni, A. M., (2005). Decentralized Sliding Mode Control Technique Based Power System Stabilizer (PSS) for Multimachine Power System, Proc. Conference on Control Applications, Toronto, Canada, August, 2005. 
Dash, P., Sahoo, N., Elangovan, S., \& Liew, A., (1996). Sliding Mode Control of a Static Controller for Synchronous Generator Stabilization, Electrical Power \& Energy Systems, Vol. 18, pp. 55-64, 1996.

DeMello, F. P. \& Concordia, C., (1969). Concepts of Synchronous Machine Stability as Affected by Excitation Control, IEEE Trans., PAS-88, Apr. 1969, 316-329.

Djukanovic, M., Khammash, M. and Vittal, V. (1998a), Application of the structured value theory for robust stability and control analysis in multimachine power systems, Part I: Framework development, IEEE, Trans. on Power Systems, Vol. 13, No. 4, November 1998.

Djukanovic, M., Khammash, M. and Vittal, V. (1998b), Application of the structured value theory for robust stability and control analysis in multimachine power systems, Part II: Numerical simulations and results, IEEE, Trans. on Power Systems, Vol. 13, No. 4, November 1998.

EPRI report, (1977). Power System Dynamic Analysis Phase I, EPRI EL-484, Project 670-1, July 1977

Huerta-Avila, H. (2005). Control no lineal robusto de sistemas eléctricos de gran escala por modos deslizante, M. Sc. Thesis, Scientific advisors: A. G. Loukianov and J. M. Cañedo, CINVESTAV, Unidad Guadalajara, México, September 2005.

Huerta-Avila, H., Loukianov, A. G., \& Cañedo, J. M. (2007a). Nested Integral Sliding Mode Control of Multimachine Power Systems, Proc. of SSSC07, Iguazu, Brazil, October 2007.

Huerta-Avila, H., Loukianov, A. G., \& Cañedo, J. M. (2007b). Nested Integral Sliding Mode Control of Large-Scale Power Systems, Proc. of CDC07, New Orleans, EUA, December 2007.

Jung, K., Kim, K., Yoon, T. \& Jang, G. (2005). Decentralized Control for Multimachine Power Systems with Nonlinear Interconnections and Disturbances, International Journal of Control, Automation, and Systems, Vol 3, No. 2 (especial edition), pp. 270-277, June 2005.

Khalil, H. K., (1996). Nonlinear systems, Prentice Hall, Inc. Simon and Schuster, New Jersey, 1996.

King, C. A., Chapman, J. W. \& Ilic, M. D., (1994). Feedback linearizing excitation control on a full scale power system model, IEEE Trans. Power Systems, 9, 1102-1109, 1994.

Kshatriya, N., Annakkage, U. D., Gole, A. M. \& Fernando, I. T., (2005). Improving the Accuracy of Normal Forma Analysis, IEEE Trans. on Power Systems, Vol. 20, No. 1, February 2005.

Liu, S., Messina, A. R., \& Vittal, V., (2006). A Normal Form Analysis Approach to Siting Power System Stabilizers (PSSs) and Assesing Power System Nonlinear Behavior, IEEE Trans. on Power Systems, Vol. 21, No. 4, November 2006.

Loukianov, A.G., (1998), Nonlinear Block Control with Sliding Mode, Automation and Remote Control, Vol.59, No.7, pp. 916-933, 1998.

Loukianov, A. G., Cañedo, J. M., Utkin, V. I. \& Cabrera-Vázquez, J., (2004). Discontinuos Controller for Power Systems: Sliding-Mode Block Control Approach, Trans. on Industrial Electronics, Vol., No. 51, No. 2, April 2004, pp. 340-353.

Loukianov, A, G., Cañedo, J. M., \& Huerta, H., (2006) Decentralized Sliding Mode Block Control of Power Systems, Proc. of PES General meeting 2006, Montreal, Quebec, Canada, June, 2006. 
Machowsky, J., Robak, S., Bialek, J. W., Bumby, J. R., \& Abi-Samra, N., (2000). Decentralized Stability-Enhancing Control of Synchronous Generator, IEEE Transactions on Power Systems, Vol. 15, No. 4, November 2000.

Mohagheghi, S., Valle, Y., Venayagamoorthy, G. K., \& Harley, R. G., (2007). A proportionalintegrator type adaptive critic design-based neurocontroller for a static compensator in a multimachine power system, IEEE, Trans. On Industrial Electronics, Vol. 54, No. 1, February, 2007.

Topalov, A.V., Cascella, G.L., Giordano, V., Cupertino, F., \& Kaynak, O., (2007). Sliding mode neuro-adaptive control of electric drives, IEEE Trans. on Industrial Electronics, vol. 54, no. 1, pp. 671-679, 2007.

Utkin, V. I., Guldner, J., \& Shi, J., (1999). Sliding Mode Control in Electromechanical Systems, Taylor \& Francis, London, 1999.

Venayagamoorthy, G. K., Harley, R. G., \& Wunsch, D. C., (2003). Dual Heuristic Programming Excitation Neruocontrol for Generators in a Multimachine Power System, IEEE Trans on Industry Applications, Vol. 39, No. 2, March/April 2003.

Wang, Y., Hill, D. J., \& Guo, G., (1998). Robust Decentralized Control for Multimachine Power Systems, IEEE Trans on Circuits and Systems-I:Fundamental Theory and Applications, Vol. 45, No. 3, March 1998, pp 271-279..

Wu, B. \& Malik, P., (2006). Multivariable Adaptive Control of Synchronous Machines in a Multimachine Power System, IEEE Trans on Power Systems, Vol. 21, No. 4, November 2006.

Xie, W., (2007). Sliding-mode-observer-based adaptive control for servo actuator with friction, IEEE Trans. on Industrial Electronics, vol. 54, no. 3, pp. 1517-1527, 2007.

Yildiz, Y., Šabanovic, A., \& Abidi, K., (2007). Sliding mode neuro-controller for uncertain systems, IEEE Trans. on Industrial Electronics, vol. 54, no. 3, pp. 1676-1685, 2007

Yousef, A. M. \& Mohamed, M. A., (2004). Multimachine Power System Stabilizer Based on Efficient Two-Layered Fuzzy Logic Controller, Transactions on Engineering and Technology V3, December 2004, pp.137-140.

\section{Appendix}

\subsection{Matrices used in generator model (1)}

$$
\begin{aligned}
& \mathbf{R}=\operatorname{diag}\left[R_{f} R_{g} R_{k d} R_{k q}-R_{s}-R_{s}\right], \mathbf{L}=\left[\begin{array}{ll}
\mathbf{L}_{11} & \mathbf{L}_{12} \\
\mathbf{L}_{21} & \mathbf{L}_{22}
\end{array}\right], \mathbf{W}(\omega)=\left[\begin{array}{cc}
0 & 0 \\
0 & I(\omega)
\end{array}\right] \in R^{6 \times 6}, \\
& I(\omega)=\left[\begin{array}{cc}
0 & -\omega \\
\omega & 0
\end{array}\right],\left[\begin{array}{ll}
\mathbf{L}_{11} & \mathbf{L}_{12} \\
\mathbf{L}_{21} & \mathbf{L}_{22}
\end{array}\right]=\left[\begin{array}{ccc}
\left(\begin{array}{cccc}
L_{f} & 0 & L_{m d} & 0 \\
0 & L_{g} & 0 & L_{m q} \\
L_{m d} & 0 & L_{k d} & 0 \\
0 & L_{m q} & 0 & L_{k q}
\end{array}\right)\left(\begin{array}{cc}
-L_{m d} & 0 \\
0 & -L_{m q} \\
-L_{m d} & 0 \\
0 & -L_{m q}
\end{array}\right) \\
\left(\begin{array}{cccc}
L_{m d} & 0 & L_{m d} & 0 \\
0 & L_{m q} & 0 & L_{m q}
\end{array}\right)\left(\begin{array}{ccc}
-L_{d} & 0 \\
0 & -L_{q}
\end{array}\right)
\end{array}\right] .
\end{aligned}
$$


$L_{d}$ and $L_{q}$ are the direct-axis and quadrature-axis self-inductances, $L_{f}$ is the field selfinductance, $L_{g}, L_{k d}$ and $L_{k q}$ are the damper windings self-inductances, $L_{m d}$ and $L_{m q}$ are the direct-axis and quadrature-axis magnetizing inductances

$\mathbf{T}=\left[\begin{array}{cc}\mathbf{I}_{4} & 0 \\ \mathbf{T}_{21} & \mathbf{T}_{22}\end{array}\right], \begin{aligned} & \mathbf{T}_{21}=-\left[\mathbf{I}_{2}-\mathbf{L}_{22}^{-1} \mathbf{L}_{21} \mathbf{L}_{11}{ }^{-1} \mathbf{L}_{12}\right]^{-1} \mathbf{L}_{22}^{-1} \mathbf{L}_{21} \mathbf{L}_{11}^{-1}, \\ & \mathbf{T}_{22}=-\left[\mathbf{I}_{2}-\mathbf{L}_{22}^{-1} \mathbf{L}_{21} \mathbf{L}_{11}{ }^{-1} \mathbf{L}_{12}\right]^{-1} \mathbf{L}_{22}^{-1},\end{aligned}, \quad$ and $\mathbf{I}_{4}$ are identity matrices of dimension 2 and 4 , respectively.

\subsection{Generators parameters}

$$
\begin{aligned}
& a_{i 1}=\frac{L_{d i}^{\prime \prime}-l_{a i}}{l_{f i}}, a_{i 2}=-\frac{L_{q i}^{\prime}-l_{a i}}{l_{g i}}, a_{i 3}=\frac{L_{d i}{ }_{d i}-l_{a i}}{l_{k d i}}, a_{i 4}=-\frac{L_{q i}{ }_{q i}-l_{a i}}{l_{k q i}}, a_{i 5}=L^{\prime \prime}{ }_{q i}-L^{\prime \prime}{ }_{d i}, \\
& b_{i 1}=-\frac{1}{\tau_{d 0 i}^{\prime}}\left(1+L_{m d i} \frac{L_{d i}^{\prime \prime}-l_{a i}}{l_{k d i} l_{f i}}\right), b_{i 2}=\frac{L_{m d i}}{\tau_{d 0 i}^{\prime}} \frac{L_{d i}^{\prime \prime}-l_{a i}}{\left(L_{d i}^{\prime}-l_{a i}\right) l_{k d i}}, b_{i 3}=-\frac{L_{m d i}}{\tau_{d 0 i}^{\prime}} \frac{L_{d i}^{\prime \prime}-l_{a i}}{L_{d i}^{\prime}-l_{a i}}, b_{i 4}=\omega_{s} \text {, } \\
& c_{i 1}=-\frac{1}{\tau_{q 0 i}^{\prime}}\left(1+\frac{L_{q i}^{\prime \prime}-l_{a i}}{l_{k q i} l_{g i}} L_{m q i}\right), c_{i 2}=\frac{L_{q}^{\prime \prime}-l_{a}}{\left(L_{q}^{\prime}-l_{a}\right) l_{k q}} \frac{L_{m q}}{\tau_{q 0}^{\prime}}, c_{i 3}=-\frac{L_{m q i}}{\tau_{q 0 i}^{\prime}}\left(\frac{L_{q i}^{\prime \prime}-l_{a i}}{L_{q i}^{\prime}-l_{a i}}\right), d_{i 1}=\frac{L_{d i}^{\prime}-l_{a i}}{\tau_{d 0 i}^{\prime \prime} l_{f i}}, \\
& d_{i 2}=-\frac{1}{\tau_{d 0 i}^{\prime \prime}}, d_{i 3}=-\frac{L_{d i}^{\prime}-l_{a i}}{\tau_{d 0 i}^{\prime \prime}}, r_{i 1}=\frac{L_{q i}^{\prime}-l_{a i}}{\tau^{\prime \prime}{ }_{q 0 i} l_{g i}}, r_{i 2}=-\frac{1}{\tau^{\prime \prime}{ }_{q 0 i}}, r_{i 3}=-\frac{L_{q i}^{\prime}-l_{a i}}{\tau_{q 0 i}^{\prime \prime}}, h_{i 1}=-\frac{\omega_{s}}{L^{\prime \prime}{ }_{d i}}, \\
& h_{i 2}=-\frac{1}{L^{\prime \prime}{ }_{d i} \tau_{d 0 i}^{\prime}}\left(1+L_{m d i} \frac{L_{d i}^{\prime}-l_{a i}}{l_{f i} l_{d d i}}\right) \frac{L_{d i}^{\prime}-l_{a i}}{l_{f i}}+\frac{1}{L^{\prime \prime}{ }_{d i} \tau^{\prime \prime}{ }_{d 0 i}} \frac{L^{\prime \prime}{ }_{d i}-l_{a i}}{L^{\prime \prime}{ }_{d i} l_{k d i}} \frac{L_{d i}^{\prime}-l_{a i}}{l_{f i}}, h_{i 4}=-\frac{1}{L^{\prime \prime}{ }_{d i}} \frac{L_{q i}^{\prime \prime}-l_{a i}}{l_{g i}}, \\
& h_{i 5}=-\frac{L_{q i}{ }_{q i}-l_{a i}}{L^{\prime \prime}{ }_{d i} l_{k q i}}, h_{i 3}=\frac{L_{m d i}}{L^{\prime \prime}{ }_{d i} \tau_{d 0 i}^{\prime}} \frac{L^{\prime \prime}{ }_{d i}-l_{a i}}{\left(L_{d i}^{\prime}-l_{a i}\right) l_{k d i}} \frac{L^{\prime \prime}{ }_{d i}-l_{a i}}{l_{f i}}-\frac{1}{L^{\prime \prime}{ }_{d i} \tau_{d 0 i}} \frac{L^{\prime \prime}{ }_{d i}-l_{a i}}{L^{\prime \prime}{ }_{d i} l_{k d i}}, h_{i 6}=\frac{L_{q i}^{\prime \prime}}{L_{d i}^{\prime \prime}}, h_{i 8}=\frac{\omega_{s}\left(L^{\prime \prime}{ }_{d i}-l_{a i}\right)}{L^{\prime \prime}{ }_{d i} l_{f i}} \text {, } \\
& k_{i 1}=-\frac{\omega_{s}}{L^{\prime \prime}{ }_{d i}}, h_{i\urcorner}=-\frac{\omega_{s} r_{a i}}{L^{\prime \prime}{ }_{d i}}-\frac{L_{m d i}}{L^{\prime \prime}{ }_{d i} \tau_{d 0 i}^{\prime}} \frac{L^{\prime \prime}{ }_{d i}-l_{a i}}{l_{f i}} \frac{L_{d i}^{\prime}-l_{a i}}{l_{f i}} \frac{L_{d i}^{\prime \prime}-l_{a i}}{L_{d i}^{\prime}-l_{a i}}-\frac{\left(L_{d i}^{\prime}-l_{a i}\right)}{L^{\prime \prime}{ }_{d i} \tau_{d 0 i}^{\prime}} \frac{L_{d i}^{\prime \prime}-l_{a i}}{L^{\prime \prime}{ }_{d i} l_{k d i}} \\
& k_{i 4}=-\frac{1}{L^{\prime \prime}{ }_{q i}} \frac{L_{q i}^{\prime \prime}{ }_{a i}}{l_{g i}} \frac{L_{q i}^{\prime}-l_{a i}}{l_{f i}}, k_{i 2}=\frac{L_{m q i}}{L^{\prime \prime} \tau_{q i}^{\prime}{ }_{q 0 i}} \frac{L^{\prime \prime}{ }_{q i}-l_{a i}}{\left(L_{q i}^{\prime}-l_{a i}\right) l_{k q i}} \frac{L_{q i}^{\prime \prime}-l_{a i}}{l_{g i}}-\frac{1}{L_{q i}^{\prime \prime} \tau_{q 0 i}^{\prime \prime}} \frac{L_{q i}^{\prime \prime}{ }_{q i}-l_{a i}}{L_{k d i}} \text {, } \\
& k_{i 5}=-\frac{1}{L_{q i}^{\prime \prime}} \frac{L_{q i}^{\prime \prime}-l_{a i}}{L_{q i} l_{k d i}}, k_{i 6}=\frac{L_{d i}^{\prime \prime}}{L_{q i}^{\prime \prime}}, \\
& k_{i 3}=-\frac{\omega_{s} r_{a i}}{L_{q i}^{\prime \prime}}-\frac{L_{m q i}}{L^{\prime \prime}{ }_{q i}^{\prime} \tau_{q 0 i}^{\prime}} \frac{L_{q i}^{\prime \prime}-l_{a i}}{l_{g i}} \frac{L_{q i}^{\prime}-l_{a i}}{l_{g i}} \frac{L_{q i}^{\prime}-l_{a i}}{L_{q i}^{\prime}-l_{a i}}-\frac{1}{L^{\prime \prime} \tau_{q i}^{\prime} \tau_{q 0 i}^{\prime}}\left(L_{q i}^{\prime}-l_{a i}\right) \frac{L_{q i}^{\prime \prime}-l_{a i}}{L^{\prime \prime} l_{q i} l_{k d i}}, \\
& k_{i 7}=-\frac{1}{L_{q i}^{\prime \prime} \tau_{q 0 i}^{\prime}}\left(1+L_{m q i} \frac{L_{q i}^{\prime}-l_{a i}}{l_{g i} l_{k q i}}\right) \frac{L_{q i}^{\prime}-l_{a i}}{l_{g i}}+\frac{1}{L_{q i}^{\prime \prime} \tau_{q 0 i}^{\prime \prime}} \frac{L_{q i}^{\prime \prime}-l_{a i}}{L_{q i}^{\prime \prime} l_{k d i}} \frac{L_{q i}^{\prime}-l_{a i}}{l_{g i}} \text {. }
\end{aligned}
$$




\begin{tabular}{|c|c|c|c|}
\hline Generator & 1 & 2 & 3 \\
\hline MVA & 247.5 & 192.0 & 128.0 \\
\hline $\mathrm{kV}$ & 16.5 & 18.0 & 13.8 \\
\hline P.F. & 1.0 & 0.85 & 0.85 \\
\hline Type & Hydro & Steam & Steam \\
\hline Speed & $180 \mathrm{r} / \mathrm{min}$ & $3600 \mathrm{r} / \mathrm{min}$ & $3600 \mathrm{r} / \mathrm{min}$ \\
\hline $\mathrm{X}_{\mathrm{d}}$ & 0.1460 & 0.8958 & 1.3125 \\
\hline $\mathrm{X}_{\mathrm{q}}$ & 0.0969 & 0.8645 & 1.2587 \\
\hline $\mathrm{X}_{\mathrm{d}}{ }^{\prime}$ & 0.0608 & 0.1198 & 0.1813 \\
\hline $\mathrm{X}_{\mathrm{q}}{ }^{\prime}$ & 0.0969 & 0.1969 & 0.2500 \\
\hline $\mathrm{\tau}_{\mathrm{d} 0}{ }^{\prime}$ & 8.9600 & 6.0000 & 5.8900 \\
\hline $\mathrm{\tau}_{\mathrm{q} 0}{ }^{\prime}$ & 0.0000 & 0.5350 & 0.6000 \\
\hline $\mathrm{X}_{\mathrm{d}}{ }^{\prime \prime}$ & 0.0400 & 0.0600 & 0.0800 \\
\hline $\mathrm{X}_{\mathrm{q}}{ }^{\prime \prime}$ & 0.0400 & 0.0600 & 0.0800 \\
\hline $\mathrm{\tau}_{\mathrm{d} 0^{\prime \prime}}$ & 0.2000 & 0.3000 & 0.4000 \\
\hline $\mathrm{\tau}_{\mathrm{q} 0}{ }^{\prime \prime}$ & 0.2000 & 0.3000 & 0.4000 \\
\hline $\mathrm{X}_{\mathrm{l}}$ & 0.0336 & 0.0521 & 0.0742 \\
\hline $\mathrm{r}_{\mathrm{a}}$ & 0.0000 & 0.0000 & 0.0000 \\
\hline $\mathrm{H}$ & 23.6400 & 6.4000 & 3.0100 \\
\hline
\end{tabular}

Table 1. Parameters of generator model (6)-(7)

\begin{tabular}{|c|c|c|c|c|c|c|c|}
\hline & Gen. 1 & Gen. 2 & Gen. 3 & & Gen. 1 & Gen. 2 & Gen. 3 \\
\hline $\mathrm{a}_{1}$ & 0.1003 & 0.1644 & 0.0945 & $\mathrm{e}_{3}$ & -5.000 & -4.0 & -2.5 \\
\hline $\mathrm{a}_{2}$ & 1.13 & 1.1787 & 0.9458 & $\mathrm{~h}_{1}$ & -1256 & -9424 & -4712 \\
\hline $\mathrm{a}_{3}$ & 0.0403 & 0.0119 & 0.0203 & $\mathrm{~h}_{2}$ & 273.4 & 863.6 & 141.3 \\
\hline $\mathrm{a}_{4}$ & 1.2552 & 1.0145 & 1.0239 & $\mathrm{~h}_{3}$ & 0.5 & -6.6 & 3.2 \\
\hline $\mathrm{a}_{5}$ & 0.020 & 0.01 & 0.010 & $\mathrm{~h}_{4}$ & -31 & -50.2 & -16.4 \\
\hline $\mathrm{b}_{1}$ & -0.017 & -0.0251 & -0.0114 & $\mathrm{~h}_{5}$ & 18.8 & 97.1 & 29.7 \\
\hline $\mathrm{b}_{2}$ & 0.522 & 2.4483 & 1.8567 & $\mathrm{~h}_{6}$ & -0.1 & -0.3 & -0.3 \\
\hline $\mathrm{b}_{3}$ & -0.5075 & -2.4185 & -1.8659 & $\mathrm{~h}_{7}$ & -4.2 & -25.4 & -12.8 \\
\hline $\mathrm{b}_{4}$ & 376.991 & 376.991 & 376.991 & $\mathrm{~h}_{8}$ & 0.1 & 1.3 & 0.9 \\
\hline $\mathrm{c}_{1}$ & -0.07 & -0.022 & -0.0472 & $\mathrm{k}_{1}$ & -1885 & -7539 & -5385 \\
\hline $\mathrm{c}_{2}$ & 0.6453 & 10.6390 & 11.4979 & $\mathrm{k}_{2}$ & 1.7 & 11.8 & 6.4 \\
\hline $\mathrm{c}_{3}$ & -0.5348 & -10.611 & -11.4581 & $\mathrm{k}_{3}$ & 5.1 & 6.9 & 34.5 \\
\hline $\mathrm{d}_{1}$ & 0.1360 & -0.2257 & 0.2267 & $\mathrm{k}_{4}$ & 31.5 & 8.37 & 39.9 \\
\hline $\mathrm{d}_{2}$ & -3.79 & -3.0659 & -2.2838 & $\mathrm{k}_{5}$ & 0.5 & 3.3 & 0.7 \\
\hline $\mathrm{d}_{3}$ & -3.33 & -3.333 & -2.5 & $\mathrm{k}_{6}$ & -5.7 & -23.6 & -13.5 \\
\hline $\mathrm{e}_{1}$ & 0.2665 & 0.5792 & 0.4395 & $\mathrm{k}_{7}$ & -0.1 & -0.8 & -1.1 \\
\hline $\mathrm{e}_{2}$ & -0.7899 & -3.2871 & -2.1290 & & & & \\
\hline
\end{tabular}

Table 2. Generators parameters 


\begin{tabular}{|l|r|r|r|l|l|l|l|}
\hline & \multicolumn{1}{|c|}{ Gen. 1 } & \multicolumn{1}{|c|}{ Gen. 2 } & Gen. 3 & & Gen. 1 & Gen. 2 & Gen. 3 \\
\hline $\mathrm{a}_{1}$ & 0.2175 & 0.0916 & 0.03 & $\mathrm{e}_{3}$ & -5.000 & -4.0 & -2.5 \\
\hline $\mathrm{a}_{2}$ & 1.1324 & 1.1787 & 0.9458 & $\mathrm{~h}_{1}$ & -1256 & -9424 & -4712 \\
\hline $\mathrm{a}_{3}$ & 0.0403 & 0.0119 & 0.0203 & $\mathrm{~h}_{2}$ & 126.1 & 1549 & 233.1 \\
\hline $\mathrm{a}_{4}$ & 1.2552 & 1.0145 & 1.0239 & $\mathrm{~h}_{3}$ & 5 & -6.8 & 3.2 \\
\hline $\mathrm{a}_{5}$ & 0.020 & 0.010 & 0.010 & $\mathrm{~h}_{4}$ & -14.5 & -100.3 & -25.8 \\
\hline $\mathrm{b}_{1}$ & -0.003 & -0.005 & -0.0023 & $\mathrm{~h}_{5}$ & 19 & 108.3 & 28.4 \\
\hline $\mathrm{b}_{2}$ & 0.1044 & 0.4897 & 0.3713 & $\mathrm{~h}_{6}$ & -0.1 & -0.3 & -0.3 \\
\hline $\mathrm{b}_{3}$ & -0.0601 & -0.0844 & -0.0358 & $\mathrm{~h}_{7}$ & -3.2 & -25.4 & -12.8 \\
\hline $\mathrm{b}_{4}$ & 376.991 & 376.991 & 376.991 & $\mathrm{~h}_{8}$ & 1 & 1.3 & 0.9 \\
\hline $\mathrm{c}_{1}$ & -0.07 & -0.022 & -0.0472 & $\mathrm{k}_{1}$ & -1885 & -7539 & -5385 \\
\hline $\mathrm{c}_{2}$ & 0.6453 & 10.6390 & 11.4979 & $\mathrm{k}_{2}$ & 1.7 & 11.8 & 6.4 \\
\hline $\mathrm{c}_{3}$ & -0.5348 & -10.611 & -11.4581 & $\mathrm{k}_{3}$ & 5.1 & 69.2 & 34.5 \\
\hline $\mathrm{d}_{1}$ & 0.1360 & -0.2257 & 0.2267 & $\mathrm{k}_{4}$ & 31.5 & 83.7 & 39.9 \\
\hline $\mathrm{d}_{2}$ & -8.2182 & -1.7090 & -1.3842 & $\mathrm{k}_{5}$ & 1.1 & 1.8 & 0.4 \\
\hline $\mathrm{d}_{3}$ & -5.0 & -3.333 & -2.5 & $\mathrm{k}_{6}$ & -5.7 & -23.6 & -13.5 \\
\hline $\mathrm{e}_{1}$ & 0.2665 & 0.5792 & 0.4395 & $\mathrm{k}_{7}$ & -0.1 & -0.8 & -1.1 \\
\hline $\mathrm{e}_{2}$ & -0.7899 & -3.2871 & -2.1290 & & & & \\
\hline
\end{tabular}

Table 3. Perturbed generators parameters

\begin{tabular}{|c|c|c|c|}
\hline & Generator 1 & Generator 2 & Generator 3 \\
\hline$k_{g i}$ & 0.02 & 0.02 & 0.03 \\
\hline$k_{0 i}$ & 7.5 & 5 & 6 \\
\hline$\rho_{2 i}$ & 8 & 10 & 9 \\
\hline$e_{1}$ & 0.9 & 0.8 & 1.2 \\
\hline$e_{2}$ & 0.01 & 0.03 & 0.02 \\
\hline$e_{3}$ & 0.001 & 0.002 & 0.001 \\
\hline
\end{tabular}

Table 4. Controllers parameters 


\subsection{Functions used in controllers design}

$$
\begin{aligned}
& f_{v i}\left(\mathbf{x}_{i}, \mathbf{i}_{i}\right)=v_{d i}\left(\mathbf{x}_{i}, \mathbf{i}_{i}\right) \varphi_{1 i}\left(\mathbf{x}_{i}, \mathbf{i}_{i}\right)+v_{q i}\left(\mathbf{x}_{i}, \mathbf{i}_{i}\right) \varphi_{2 i}\left(\mathbf{x}_{i}, \mathbf{i}_{i}\right), b_{v i}=\left(\begin{array}{l}
\left.h_{i 2} b_{i 4}+\frac{1}{k_{i 1}} k_{i 4} b_{i 4} x_{2 i}\right) \\
h_{1 i}\left(\mathbf{x}_{i}, \mathbf{i}_{i}\right)=-\frac{1}{h_{i 1}}\left[\begin{array}{l}
h_{i 2}\left(b_{i 1} x_{3 i}+b_{i 2} x_{5 i}+b_{i 3} i_{d i}\right)+h_{i 3}\left(d_{i 1} x_{3 i}+d_{i 2} x_{5 i}+d_{i 3} i_{1 i}\right)+ \\
h_{i 4}\left(\begin{array}{l}
x_{2 i}\left(c_{i 1} x_{4 i}+c_{i 2} x_{6 i}+c_{i 3} i_{2 i}\right)+ \\
\left(f_{\omega i}\left(\mathbf{x}_{i}, \mathbf{i}_{i}, T_{m i}\right)-q_{i}\left(\mathbf{x}_{i}, \mathbf{i}_{i}\right) x_{3 i}\right) x_{4 i}
\end{array}\right)+h_{i 5}\left(\begin{array}{l}
x_{2 i}\left(r_{i 1} x_{4 i}+r_{i 2} x_{6 i}+r_{i 3} i_{2 i}\right)+ \\
\left(f_{\omega i}\left(\mathbf{x}_{i}, \mathbf{i}_{i}, T_{m i}\right)-q_{i}\left(\mathbf{x}_{i}, \mathbf{i}_{i}\right) x_{3 i}\right) x_{6 i}
\end{array}\right)
\end{array}\right], \\
\varphi_{2 i}\left(\mathbf{x}_{i}, \mathbf{i}_{i}\right)=-\frac{1}{k_{i 1}}\left[\begin{array}{l}
k_{i 2} \dot{x}_{4 i}+k_{i 3}\left(r_{i 1} x_{4 i}+r_{i 2} x_{6 i}+r_{i 3} i_{2 i}\right)+ \\
k_{i 4}\left(x_{2 i}\left(b_{i 1} x_{3 i}+b_{i 2} x_{5 i}+b_{i 3} i_{1 i}\right)+\left(f_{\omega i}\left(\mathbf{x}_{i}, \mathbf{i}_{i}, T_{m i}\right)-q_{i}\left(\mathbf{x}_{i}, \mathbf{i}_{i}\right) x_{3 i}\right) x_{3 i}\right) \\
+k_{i 5}\left(x_{2 i}\left(d_{i 1} x_{3 i}+d_{i 2} x_{5 i}+d_{i 3} i_{1 i}\right)+\left(f_{\omega i}\left(\mathbf{x}_{i}, \mathbf{i}_{i}, T_{m i}\right)-q_{i}\left(\mathbf{x}_{i}, \mathbf{i}_{i}\right) x_{3 i}\right) x_{5 i}\right)
\end{array}\right], \\
g_{v i}\left(\mathbf{x}_{i}, \mathbf{i}_{i}\right)=h_{i 6} \frac{d}{d t} i_{d i}+h_{i 7}\left(x_{2 i} \frac{d}{d t} i_{q i}+\dot{x}_{2 i} i_{q i}\right)+k_{i 6}\left(x_{2 i} \frac{d}{d t} i_{d i}+\dot{x}_{2 i}\right)+k_{i 7} \frac{d}{d t} i_{q i}+h_{i 8} \frac{d}{d t} v_{f i}+\Delta f_{v i} .
\end{array}\right.
\end{aligned}
$$




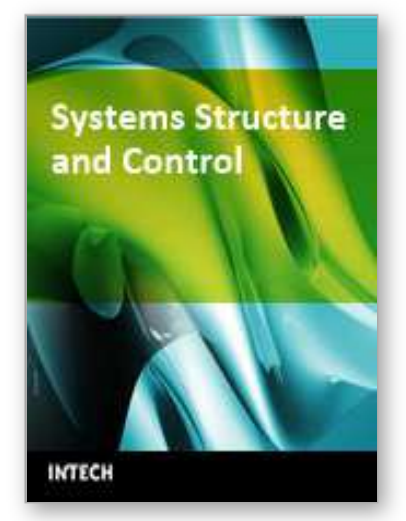

\author{
Systems Structure and Control \\ Edited by Petr Husek
}

ISBN 978-953-7619-05-3

Hard cover, 248 pages

Publisher InTech

Published online 01, August, 2008

Published in print edition August, 2008

The title of the book System, Structure and Control encompasses broad field of theory and applications of many different control approaches applied on different classes of dynamic systems. Output and state feedback control include among others robust control, optimal control or intelligent control methods such as fuzzy or neural network approach, dynamic systems are e.g. linear or nonlinear with or without time delay, fixed or uncertain, onedimensional or multidimensional. The applications cover all branches of human activities including any kind of industry, economics, biology, social sciences etc.

\title{
How to reference
}

In order to correctly reference this scholarly work, feel free to copy and paste the following:

Hector Huerta, Alexander Loukianov and Jose M. Canedo (2008). Integral Sliding Modes with Block Control of Multimachine Electric Power Systems, Systems Structure and Control, Petr Husek (Ed.), ISBN: 978-953-761905-3, InTech, Available from:

http://www.intechopen.com/books/systems_structure_and_control/integral_sliding_modes_with_block_control_ of_multimachine_electric_power_systems

\section{INTECH}

open science | open minds

\section{InTech Europe}

University Campus STeP Ri

Slavka Krautzeka 83/A

51000 Rijeka, Croatia

Phone: +385 (51) 770447

Fax: +385 (51) 686166

www.intechopen.com

\section{InTech China}

Unit 405, Office Block, Hotel Equatorial Shanghai

No.65, Yan An Road (West), Shanghai, 200040, China

中国上海市延安西路65号上海国际贵都大饭店办公楼405单元

Phone: +86-21-62489820

Fax: +86-21-62489821 
(C) 2008 The Author(s). Licensee IntechOpen. This chapter is distributed under the terms of the Creative Commons Attribution-NonCommercialShareAlike-3.0 License, which permits use, distribution and reproduction for non-commercial purposes, provided the original is properly cited and derivative works building on this content are distributed under the same license. 